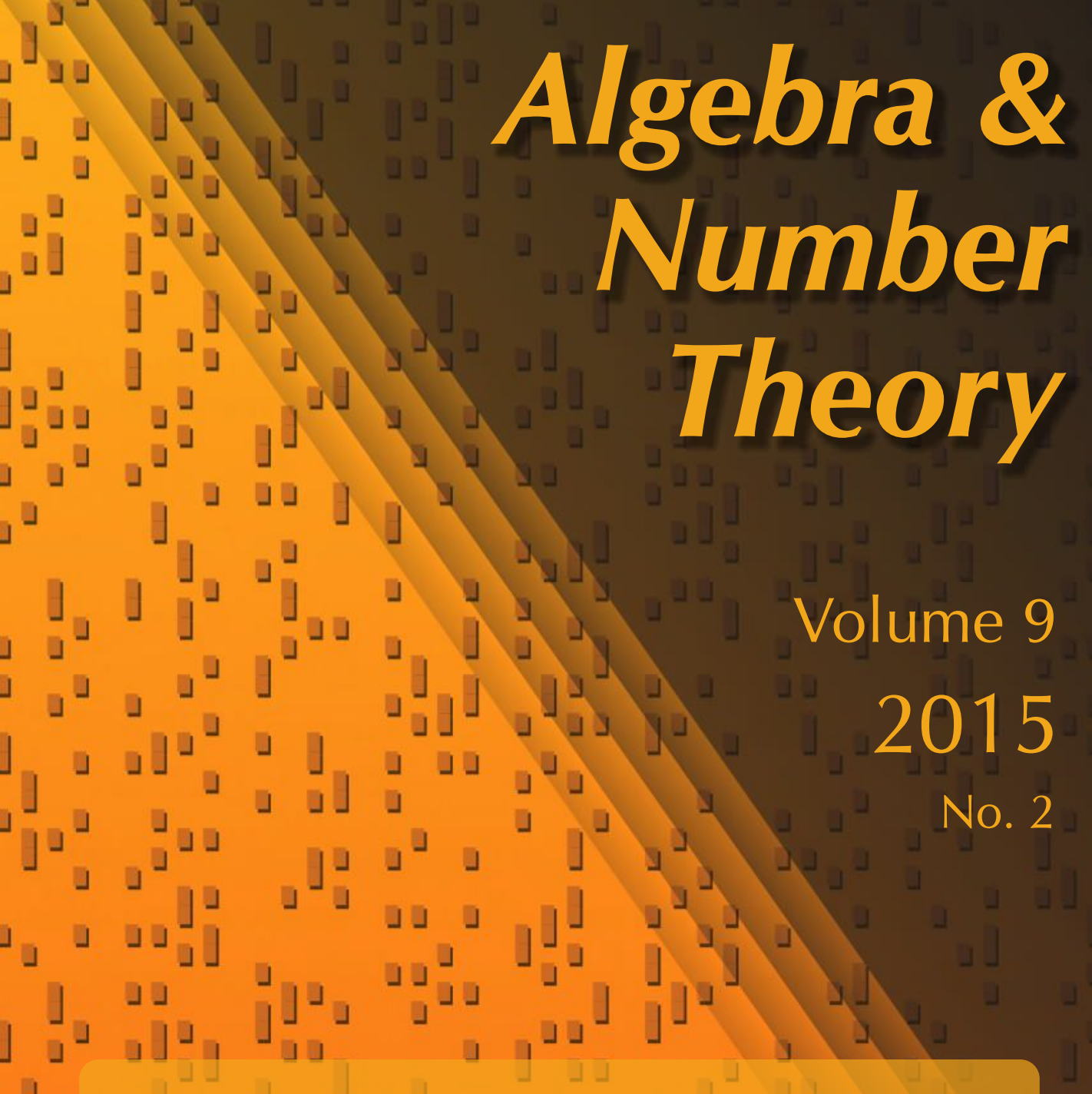

Semistable periods of finite slope families

Ruochuan Liu

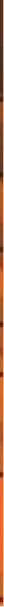




\title{
Semistable periods of finite slope families
}

\author{
Ruochuan Liu
}

\begin{abstract}
We introduce the notion of finite slope families to encode the local properties of the $p$-adic families of Galois representations appearing in the work of Harris, Lan, Taylor and Thorne on the construction of Galois representations for (nonself-dual) regular algebraic cuspidal automorphic representations of GL $(n)$ over $\mathrm{CM}$ fields. Our main result is to prove the analytic continuation of semistable (and crystalline) periods for such families.
\end{abstract}

$\begin{array}{lr}\text { Introduction and notation } & 435 \\ \text { Notation } & 438 \\ \text { 1. Families of }(\varphi, \Gamma) \text {-modules } & 439 \\ \text { 2. Cohomology of families of }(\varphi, \Gamma) \text {-modules } & 441 \\ \text { 3. Families of Hodge-Tate }(\varphi, \Gamma) \text {-modules } & 444 \\ \text { 4. Families of de Rham }(\varphi, \Gamma) \text {-modules } & 447 \\ \text { 5. p-adic local monodromy for families of de Rham }(\varphi, \Gamma) \text {-modules } & 451 \\ \text { 6. Proof of the main theorem } & 454 \\ \text { Acknowledgements } & 457 \\ \text { References } & 457\end{array}$

\section{Introduction and notation}

Skinner and Urban [2006], in their ICM talk, outlined a program to connect the order of vanishing of the $L$-functions of certain polarized regular motives with the rank of the associated Bloch-Kato Selmer groups. Their strategy is to deform those motives along certain $p$-adic families, the so-called eigenfamilies, to construct the expected extensions. To this end, they introduced the notion of finite slope families of $p$-adic representations to encode the local properties of the Galois representations arising from those $p$-adic families. One may view the finite slope families as a generalization of the $p$-adic families of Galois representations arising from the Coleman-Mazur eigencurve. Bellaïche and Chenevier [2009] introduced the notion of weakly refined families of $p$-adic representations to encode the local properties of the latter. More precisely, a family of weakly refined $p$-adic representations is

Keywords: finite slope families, semistable, $(\varphi, \Gamma)$-modules. 
a family of $p$-adic representations ${ }^{1}$ over a rigid analytic space with a Zariski-dense subset of crystalline points which have crystalline periods of a prescribed Frobenius eigenvalue and constant Hodge-Tate weight. Moreover, this constant weight is the largest one ${ }^{2}$ of all Hodge-Tate weights, and the difference of this constant weight with any other weight is unbounded over the base. For example, in the case of the eigencurve, one can take the subset of all classical eigenforms, and the prescribed Frobenius eigenvalue and constant Hodge-Tate weight are the function of $U_{p}$-eigenvalues and 0 respectively. On the other hand, finite slope families generalize weakly refined families in the way that allows multiple prescribed Frobenius eigenvalues and constant Hodge-Tate weights. Skinner and Urban then used the (unproven) analytic continuation of crystalline periods of finite slope families to deduce that the extensions constructed by $p$-adic deformations lie in the Selmer groups.

Most recently, Harris, Lan, Taylor and Thorne [Harris et al. 2014] (and Scholze independently) constructed Galois representations for (non-self-dual) regular algebraic cuspidal automorphic representations of GL $(n)$ over CM fields. It turns out that these Galois representations emerge from certain $p$-adic families whose local properties generalize Skinner and Urban's finite slope families by allowing prescribed semistable periods. Therefore, to show that the Galois representations constructed by Harris, Lan, Taylor and Thorne have the expected properties at $p$, one needs to show the analytic continuation of semistable periods for those $p$-adic families.

In this paper, we make use of the notion of finite slope families to encode the local properties of the $p$-adic families of Galois representations appearing in the work of Harris, Lan, Taylor and Thorne; this generalizes the original definition of Skinner and Urban. Our main result is then to prove the analytic continuation of semistable periods for such families. This will provide a necessary ingredient in Skinner and Urban's ICM program. Besides, we recently learned from Taylor that Ila Varma, as part of an ongoing project, will establish the expected properties of those Galois representations based on the results of this paper. We also note that recently Shah [2013] proved some results about interpolating Hodge-Tate and de Rham periods in families of $p$-adic Galois representations which may be applied to some related situations.

In the following, we state our main results precisely. We fix a finite extension $K$ of $\mathbb{Q}_{p}$. Let $K_{0}$ be the maximal unramified subextension of $K$, and let $f=\left[K_{0}: \mathbb{Q}_{p}\right]$. We also fix a finite extension $F$ of $\mathbb{Q}_{p}$ contained in $\overline{\mathbb{Q}}_{p}$ such that $\operatorname{Hom}(K, F)=$ $\operatorname{Hom}\left(K, \overline{\mathbb{Q}}_{p}\right)$; here Hom denotes the set of $\mathbb{Q}_{p}$-algebra homomorphisms.

\footnotetext{
${ }^{1}$ Strictly speaking, Bellaïche-Chenevier used pseudorepresentations rather than genuine representations in their definition of weakly refined families.

${ }^{2}$ We normalize the Hodge-Tate weight such that the $p$-adic cyclotomic character has Hodge-Tate weight 1.
} 
Definition 0.1. Let $X$ be a reduced rigid analytic space over $F$. A finite slope family of $p$-adic representations of dimension $d$ over $X$ is a locally free coherent $O_{X}$-module $V_{X}$ of rank $d$ equipped with a continuous $G_{K}$-action, together with the following data:

(1) Positive integers $b, c$.

(2) A monic polynomial $Q(T) \in \mathscr{O}_{X}(X)[T]$ of degree $m$ with unit constant term.

(3) A subset $Z$ of $X$ such that, for all $z$ in $Z, V_{z}$ is semistable with nonpositive Hodge-Tate weights, and, for all $B \in \mathbb{Z}$, the set of $z$ in $Z$ such that $V_{z}$ has $d-c$ Hodge-Tate weights less than $B$ is Zariski-dense in $X$.

(4) For $z \in Z$, a $K_{0} \otimes_{\mathbb{Q}_{p}} k(z)$-direct summand $\mathscr{F}_{z}$ of $D_{\text {st }}^{+}\left(V_{z}\right)$ which is free of rank $c$ and stable under $\varphi$ and $N$ such that $\varphi^{f}$ has characteristic polynomial $Q(z)(T)$ and all Hodge-Tate weights of $\mathscr{F}_{z}$ lie in $[-b, 0]$.

We also need to extend the functors $D_{\text {crys }}^{+}$and $D_{\text {st }}^{+}$to families of $p$-adic representations over rigid analytic spaces.

Definition 0.2. Let $X$ be a rigid analytic space over $\mathbb{Q}_{p}$, and let $V_{X}$ be locally free coherent $O_{X}$-module equipped with a continuous $G_{K}$-action. Define $D_{\text {crys }}^{+}\left(V_{X}\right)$ and $D_{\text {st }}^{+}\left(V_{X}\right)$ to be the presheaves

$$
M(S) \mapsto D_{\text {crys }}^{+}\left(V_{S}\right)=\left(V_{S} \widehat{\otimes}_{\mathbb{Q}_{p}} B_{\text {crys }}^{+}\right)^{G_{K}}
$$

and

$$
M(S) \mapsto D_{\mathrm{st}}^{+}\left(V_{S}\right)=\left(V_{S} \hat{\otimes}_{\mathbb{Q}_{p}} \boldsymbol{B}_{\mathrm{st}}^{+}\right)^{G_{K}},
$$

respectively, where $M(S)$ runs through all admissible affinoid subdomain of $X$; here $V_{S}$ is the restriction of $V_{X}$ on $M(S)$.

Now we can state our main result precisely:

Theorem 0.3. Let $V_{X}$ be a finite slope family over $X$. Then there exists a surjective proper morphism $X^{\prime} \rightarrow X$ so that $\left(K \otimes_{K_{0}} D_{\mathrm{st}}^{+}\left(V_{X^{\prime}}\right)\right)^{Q\left(\varphi^{f}\right)=0}$ has a rank-c locally free coherent $K_{0} \otimes_{\mathbb{Q}_{p}} O_{X^{\prime}}$-submodule which specializes to a rank-c free $K_{0} \otimes_{\mathbb{Q}_{p}} k(x)$-submodule in $D_{\mathrm{st}}^{+}\left(V_{x}\right)$ for any $x \in X^{\prime}$. As a consequence, for any $x \in X, D_{\mathrm{st}}^{+}\left(V_{x}\right)^{Q(x)\left(\varphi^{f}\right)=0}$ has a free $K_{0} \otimes_{\mathbb{Q}_{p}} k(x)$-submodule of rank $c$.

The next result follows immediately:

Corollary 0.4. Let $V_{X}$ be a finite slope family over $X$. If $V_{z}$ is crystalline for any $z \in Z$, then there exists a surjective proper morphism $X^{\prime} \rightarrow X$ so that $\left(K \otimes_{K_{0}} D_{\text {crys }}^{+}\left(V_{X^{\prime}}\right)\right)^{Q\left(\varphi^{f}\right)=0}$ has a rank-c locally free coherent $K_{0} \otimes_{\mathbb{Q}_{p}} \mathcal{O}_{X^{\prime}-}$ submodule which specializes to a rank-c free $K_{0} \otimes_{\mathbb{Q}_{p}} k(x)$-submodule in $D_{\text {crys }}^{+}\left(V_{x}\right)$ for every $x \in X^{\prime}$. As a consequence, $D_{\text {crys }}^{+}\left(V_{x}\right)^{Q(x)\left(\varphi^{f}\right)=0}$ has a free $K_{0} \otimes_{\mathbb{Q}_{p}} k(x)$ submodule of rank $c$ for every $x \in X$. 
Since the families of $p$-adic representations arising from the Coleman-Mazur eigencurve are special cases of finite slope families, Theorem 0.3 generalizes the famous result of Kisin [2003] on the analytic continuation of crystalline periods over the eigencurve. However, even in the case that the prescribed periods are crystalline, our method is completely different from his. In fact, in the work of Kisin as well as our recent enhancement [Liu 2014], one crucially uses the fact that the families of $p$-adic representations arising from the eigencurve have only one constant Hodge-Tate weight. On the other hand, our strategy and techniques are largely inspired by the work of Berger and Colmez [2008] on families of de Rham representations with bounded Hodge-Tate weights, and Kedlaya, Pottharst and Xiao [Kedlaya et al. 2014] on the cohomology of families of $(\varphi, \Gamma)$-modules. In fact, for a finite slope family, we first construct a subfamily of $(\varphi, \Gamma)$-modules interpolating the prescribed semistable periods, after making a proper and surjective base change. This is achieved by adapting some techniques of [Kedlaya et al. 2014]. This subfamily of $(\varphi, \Gamma)$-modules is expected to be semistable and produce the desired semistable periods. However, we are unable to prove this directly due to some technical obstacles. Instead, we first show that this subfamily of $(\varphi, \Gamma)$ modules is de Rham, using the fact that it is de Rham at a Zariski-dense subset of the base. To this end, we develop a theory of families of Hodge-Tate and de Rham $(\varphi, \Gamma)$-modules with bounded Hodge-Tate weights, which generalizes the theory of families of Hodge-Tate and de Rham representations with bounded HodgeTate weights developed in [Berger and Colmez 2008]. Then we prove the $p$-adic local monodromy for the restrictions of families of de Rham $(\varphi, \Gamma)$-modules with bounded Hodge-Tate weights on their Shilov boundaries by mimicking the proof for families of de Rham representations with bounded Hodge-Tate weights given in [loc. cit.]. This implies that the de Rham periods of this subfamily of $(\varphi, \Gamma)$ modules become potentially semistable after restricting on the Shilov boundary. Finally, we use a key lemma due to Berger and Colmez [2008] to conclude that these de Rham periods are actually semistable.

\section{Notation}

We choose a compatible sequence of primitive $p$-power roots of unity $\left(\varepsilon_{n}\right)_{n \geq 0}$, i.e., each $\varepsilon_{n} \in \overline{\mathbb{Q}}_{p}$ is a primitive $p^{n}$-th root of 1 , and they satisfy $\varepsilon_{n+1}^{p}=\varepsilon_{n}$ for all $n \geq 0$. Fix $\varepsilon=\left(\varepsilon_{0}, \varepsilon_{1}, \ldots\right)$, and let $t=\log [\varepsilon]$ be Fontaine's $p$-adic $2 \pi i$. For a finite extension $L$ of $\mathbb{Q}_{p}$ in $\mathbb{C}_{p}$, let $L_{n}=L\left(\varepsilon_{n}\right)$ for $n \geq 1$, and let $L_{\infty}=\bigcup_{n \in \mathbb{N}} L_{n}$. Let $L_{0}^{\prime}$ be the maximal unramified extension of $\mathbb{Q}_{p}$ in $L_{\infty}$. Let $\Gamma_{L}=\operatorname{Gal}\left(L_{\infty} / L\right)$ and $\Gamma_{L_{n}}=\operatorname{Gal}\left(L_{\infty} / L_{n}\right)$ for $n \geq 1$. For simplicity, denote $\Gamma_{K}$ and $\Gamma_{K_{n}}$ by $\Gamma$ and $\Gamma_{n}$ respectively. Let $\chi$ denote the $p$-adic cyclotomic character. For a $p$ adic representation $V$ of $G_{K}$ and $n \in \mathbb{Z}$, we set $V(n)=V \otimes \chi^{n}$. For $n \geq 0$, let $r_{n}=p^{n-1}(p-1)$. For $s>0$, let $n(s)$ be the maximal integer $n$ such that $r_{n} \leq s$. 


\section{Families of $(\varphi, \Gamma)$-modules}

In this section we recall the notion of families of $(\varphi, \Gamma)$-modules over rigid analytic spaces. For the period rings involved in this paper, we follow the notation introduced in [Berger 2002], and we refer the reader to that paper for precise definitions. Note that this is different from the "Robba ring" type notation used in [Kedlaya et al. 2014]. A good dictionary for these two types of notation is given in [Berger 2008a, §1].

Definition 1.1. Let $A$ be a Banach algebra over $\mathbb{Q}_{p}$. For $s>0$, a $\varphi$-module over $\boldsymbol{B}_{\mathrm{rig}, K}^{\dagger, s} \hat{\otimes}_{\mathbb{Q}_{p}} A$ is a finite projective $\boldsymbol{B}_{\mathrm{rig}, K}^{\dagger, s} \widehat{\otimes}_{\mathbb{Q}_{p}} A$-module $D_{A}^{s}$ equipped with an isomorphism

$$
\varphi^{*} D_{A}^{s} \cong D_{A}^{s} \otimes_{\boldsymbol{B}_{\mathrm{rig}, K}^{\dagger, s} \hat{\otimes}_{\mathbb{Q}_{p}} A} \boldsymbol{B}_{\mathrm{rig}, K}^{\dagger, p s} \widehat{\otimes}_{\mathbb{Q}_{p}} A .
$$

A $\varphi$-module $D_{A}$ over $\boldsymbol{B}_{\mathrm{rig}, K}^{\dagger} \hat{\otimes}_{\mathbb{Q}_{p}} A$ is the base change to $\boldsymbol{B}_{\text {rig, } K}^{\dagger} \hat{\otimes}_{\mathbb{Q}_{p}} A$ of a $\varphi$-module $D_{A}^{s}$ over $\boldsymbol{B}_{\text {rig, } K}^{\dagger, s} \hat{\otimes}_{\mathbb{Q}_{p}} A$ for some $s>0$. A $(\varphi, \Gamma)$-module over $\boldsymbol{B}_{\text {rig, }, K}^{\dagger, s} \hat{\otimes}_{\mathbb{Q}_{p}} A$ is a $\varphi$-module $D_{A}^{s}$ over $\boldsymbol{B}_{\text {rig, } K}^{\dagger, s} \widehat{\otimes}_{\mathbb{Q}_{p}} A$ equipped with a commuting $\boldsymbol{B}_{\text {rig, }, K^{\dagger}}^{\dagger, s e m i l i n e a r}$ and $A$-linear continuous action of $\Gamma$. A $(\varphi, \Gamma)$-module $D_{A}$ over $\boldsymbol{B}_{\text {rig, } K}^{\dagger} \widehat{\otimes}_{\mathbb{Q}_{p}} A$ is the base change to $\boldsymbol{B}_{\mathrm{rig}, K}^{\dagger} \hat{\otimes}_{\mathbb{Q}_{p}} A$ of a $(\varphi, \Gamma)$-module $D_{A}^{s}$ over $\boldsymbol{B}_{\mathrm{rig}, K}^{\dagger, s} \hat{\otimes}_{\mathbb{Q}_{p}} A$ for some $s>0$.

Notation 1.2. For a morphism $A \rightarrow B$ of Banach algebras over $\mathbb{Q}_{p}$, we denote by $D_{B}^{s}$ and $D_{B}$ the base changes of $D_{A}^{s}$ and $D_{A}$ to $\boldsymbol{B}_{\mathrm{rig}, K}^{\dagger, s} \hat{\otimes}_{\mathbb{Q}_{p}} B$ and $\boldsymbol{B}_{\mathrm{rig}, K}^{\dagger} \hat{\otimes}_{\mathbb{Q}_{p}} B$, respectively. When $A=S$ is an affinoid algebra over $\mathbb{Q}_{p}$ and $x \in M(S)$, we denote $D_{k(x)}^{s}$ and $D_{k(x)}$ by $D_{x}^{s}$ and $D_{x}$ instead.

To define $(\varphi, \Gamma)$-modules over general rigid analytic spaces, one needs to show that $\varphi$-modules over affinoid spaces satisfy the gluing property. To this end, we recall the notion of $\varphi$-bundles introduced in [Kedlaya et al. 2014]. Let $S$ be an affinoid algebra over $\mathbb{Q}_{p}$. For $0<s_{1}<s_{2}$ which are sufficiently large, a vector bundle over $\boldsymbol{B}_{K}^{\left[s_{1}, s_{2}\right]} \hat{\otimes}_{\mathbb{Q}_{p}} S$ is a finite projective module $D_{S}^{\left[s_{1}, s_{2}\right]}$ over $\boldsymbol{B}_{K}^{\left[s_{1}, s_{2}\right]} \hat{\otimes}_{\mathbb{Q}_{p}} S$. By the identification of $\boldsymbol{B}_{K}^{\left[s_{1}, s_{2}\right]}$ with the ring of rigid analytic functions over the closed annulus $s_{1} \leq v_{p}(T) \leq s_{2}$ over $K_{0}^{\prime}$, one may identify $D_{S}^{\left[s_{1}, s_{2}\right]}$ with a locally free coherent sheaf over the product of the annulus $s_{1} \leq v_{p}(T) \leq s_{2}$ over $K_{0}^{\prime}$ with $M(S)$ in the category of rigid analytic spaces over $\mathbb{Q}_{p}$. It then follows that vector bundles over $\boldsymbol{B}_{K}^{\left[s_{1}, s_{2}\right]} \hat{\otimes}_{\mathbb{Q}_{p}} S$ satisfy the gluing property for the weak $G$-topology of $M(S)$. For sufficiently large $s$, a vector bundle over $\boldsymbol{B}_{\mathrm{rig}, K}^{\dagger, s} \hat{\otimes}_{\mathbb{Q}_{p}} S$ consists of one vector bundle $D_{S}^{\left[s_{1}, s_{2}\right]}$ over each ring $\boldsymbol{B}_{K}^{\left[s_{1}, s_{2}\right]} \hat{\otimes}_{\mathbb{Q}_{p}} S$ with $s \leq s_{1} \leq s_{2}$, together with isomorphisms

$$
D_{S}^{\left[s_{1}, s_{2}\right]} \otimes_{B_{K}^{\left[s_{1}, s_{2}\right]} \hat{\otimes}_{\mathbb{Q}_{p}} S} \boldsymbol{B}_{K}^{\left[s_{1}^{\prime}, s_{2}^{\prime}\right]} \hat{\otimes}_{\mathbb{Q}_{p}} S \cong D_{S}^{\left[s_{1}^{\prime}, s_{2}^{\prime}\right]}
$$

for all $s \leq s_{1}^{\prime} \leq s_{1} \leq s_{2} \leq s_{2}^{\prime}$ satisfying the cocycle conditions. A $\varphi$-bundle over $\boldsymbol{B}_{\text {rig, } K}^{\dagger, s} \hat{\otimes}_{\mathbb{Q}_{p}} S$ is a vector bundle $\left(D_{S}^{\left[s_{1}, s_{2}\right]}\right)_{s \leq s_{1} \leq s_{2}}$ over $\boldsymbol{B}_{\text {rig, } K}^{\dagger, s} \hat{\otimes}_{\mathbb{Q}_{p}} S$ equipped with

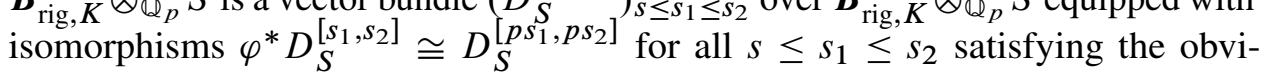
ous compatibility conditions. When $s$ is sufficiently large, by [Kedlaya et al. 
2014, Proposition 2.2.7], the natural functor from the category of $\varphi$-modules over $\boldsymbol{B}_{\text {rig, } K}^{\dagger, s} \hat{\otimes}_{\mathbb{Q}_{p}} S$ to the category of $\varphi$-bundles over $\boldsymbol{B}_{\text {rig, } K}^{\dagger, s} \hat{\otimes}_{\mathbb{Q}_{p}} S$ is an equivalence of categories. Note that by the gluing property of vector bundles, one can glue $\varphi$-bundles $\boldsymbol{B}_{\mathrm{rig}, K}^{\dagger, s} \hat{\otimes}_{\mathbb{Q}_{p}} S$ over $M(S)$. Therefore this equivalence of categories enables us to glue $\varphi$-modules over affinoid spaces.

Definition 1.3. Let $X$ be a rigid analytic space over $\mathbb{Q}_{p}$. A family of $(\varphi, \Gamma)$-modules $D_{X}$ over $X$ is a compatible family of $(\varphi, \Gamma)$-modules $D_{S}$ over $\boldsymbol{B}_{\mathrm{rig}, K}^{\dagger} \hat{\otimes}_{\mathbb{Q}_{p}} S$ for each affinoid subdomain $M(S)$ of $X$. By the gluing property of $\varphi$-modules over affinoid spaces, one may view $D_{X}$ as a sheaf over $X$ for the weak $G$-topology (which hence extends uniquely to the strong $G$-topology).

Theorem 1.4. Let $A$ be a Banach algebra over $\mathbb{Q}_{p}$, and let $V_{A}$ be a finite locally free A-linear representation of $G_{K}$. Then there is a $(\varphi, \Gamma)$-module $\mathrm{D}_{\mathrm{rig}}^{\dagger}\left(V_{A}\right)$ over $\boldsymbol{B}_{\mathrm{rig}, K}^{\dagger} \hat{\otimes}_{\mathbb{Q}_{p}}$ A functorially associated to $V_{A}$. The rule $V_{A} \mapsto \mathrm{D}_{\text {rig }}^{\dagger}\left(V_{A}\right)$ is fully faithful and exact, and it commutes with base change in $A$.

Proof. See [Kedlaya and Liu 2010, Theorem 3.11], which generalizes [Berger and Colmez 2008, Théorème 4.2.9]. Note that both the results do not really verify the $\varphi$-module condition. This gap is fixed by [Liu 2014, Theorem 1.1.4].

Let $A$ be a Banach algebra over $K_{0}$. Recall that one has a canonical decomposition

$$
A \otimes_{\mathbb{Q}_{p}} K_{0} \cong \prod_{\sigma \in \operatorname{Gal}\left(K_{0} / \mathbb{Q}_{p}\right)} A_{\sigma},
$$

where each $A_{\sigma}$ is the base change of $A$ by the automorphism $\sigma$. Furthermore, the $\operatorname{Gal}\left(K_{0} / \mathbb{Q}_{p}\right)$-action permutes all the $A_{\sigma}$ such that $\tau\left(A_{\sigma}\right)=A_{\tau \sigma}$. For any $a \in A^{\times}$, we equip $A \otimes_{\mathbb{Q}_{p}} K_{0}$ with a $1 \otimes \varphi$-semilinear action $\varphi$ by setting

$$
\varphi\left(\left(x_{1}, x_{\varphi}, \ldots, x_{\varphi f-1}\right)\right)=\left(a x_{\varphi f-1}, x_{1}, \ldots, x_{\varphi f-2}\right),
$$

where $\varphi$ is the geometric Frobenius and $x_{\varphi^{i}} \in A_{\varphi^{i}}$ for each $0 \leq i \leq f-1$; we denote this $\varphi$-module by $D_{a}$. It is clear that the $\varphi$-action on $D_{a}$ satisfies $\varphi^{f}=1 \otimes a$.

We fix a uniformizer $\pi_{K}$ of $K$.

Definition 1.5. For any continuous character $\delta: K^{\times} \rightarrow A^{\times}$, we define a rank-1 $(\varphi, \Gamma)$-module $\left(\boldsymbol{B}_{\mathrm{rig}, K}^{\dagger} \hat{\otimes}_{\mathbb{Q}_{p}} A\right)(\delta)$ over $\boldsymbol{B}_{\mathrm{rig}, K}^{\dagger} \hat{\otimes}_{\mathbb{Q}_{p}} A$ as follows. If $\left.\delta\right|_{\mathcal{O}_{K}^{\times}}=1$, we set

$$
\left(\boldsymbol{B}_{\mathrm{rig}, K}^{\dagger} \hat{\otimes}_{\mathbb{Q}_{p}} A\right)(\delta)=\left(\boldsymbol{B}_{\mathrm{rig}, K}^{\dagger} \hat{\otimes}_{\mathbb{Q}_{p}} A\right) \otimes_{A \otimes_{\mathbb{Q}_{p}} K_{0}} D_{\delta\left(\pi_{K}\right)},
$$

where we equip $D_{\delta\left(\pi_{K}\right)}$ with the trivial $\Gamma$-action. For general $\delta$, we write $\delta=\delta^{\prime} \delta^{\prime \prime}$ such that $\delta^{\prime}\left(\pi_{K}\right)=1$ and $\left.\delta^{\prime \prime}\right|_{O_{K}^{\times}}=$id. We view $\delta^{\prime}$ as an $A$-valued character of $W_{K}$ via the local reciprocity map, and extend it to a character of $G_{K}$ continuously. We then set

$$
\left(\boldsymbol{B}_{\mathrm{rig}, K}^{\dagger} \hat{\otimes}_{\mathbb{Q}_{p}} A\right)(\delta)=\mathrm{D}_{\text {rig }}^{\dagger}\left(\delta^{\prime}\right) \otimes_{\boldsymbol{B}_{\mathrm{rig}, K}^{\dagger} \hat{\otimes}_{\mathbb{Q}_{p}} A}\left(\boldsymbol{B}_{\text {rig }, K}^{\dagger} \hat{\otimes}_{\mathbb{Q}_{p}} A\right)\left(\delta^{\prime \prime}\right) .
$$


For any $(\varphi, \Gamma)$-module $D_{A}$ over $\boldsymbol{B}_{\text {rig, } K}^{\dagger} \hat{\otimes}_{\mathbb{Q}_{p}} A$, put

$$
D_{A}(\delta)=D_{A} \otimes_{\boldsymbol{B}_{\mathrm{rig}, K}^{\dagger} \hat{\otimes}_{\mathbb{Q}_{p}} A}\left(\boldsymbol{B}_{\mathrm{rig}, K}^{\dagger} \hat{\otimes}_{\mathbb{Q}_{p}} A\right)(\delta) .
$$

Let $X$ be a rigid analytic space over $\mathbb{Q}_{p}$. For a continuous character $\delta: K^{\times} \rightarrow$ $\mathcal{O}(X)^{\times}$and a family of $(\varphi, \Gamma)$-module $D_{X}$ over $X$, we define the families of $(\varphi, \Gamma)$ modules $\left(\boldsymbol{B}_{\mathrm{rig}, K}^{\dagger} \hat{\otimes}_{\mathbb{Q}_{p}} \mathcal{O}_{X}\right)(\delta)$ and $D_{X}(\delta)$ by gluing $\left(\boldsymbol{B}_{\mathrm{rig}, K}^{\dagger} \hat{\otimes}_{\mathbb{Q}_{p}} S\right)(\delta)$ and $D_{S}(\delta)$ for all affinoid subdomains $M(S)$, respectively.

\section{Cohomology of families of $(\varphi, \Gamma)$-modules}

Let $\Delta_{K}$ be the $p$-torsion subgroup of $\Gamma$. Choose $\gamma_{K}$ in $\Gamma_{K}$ whose image in $\Gamma / \Delta_{K}$ is a topological generator.

Definition 2.1. For a $(\varphi, \Gamma)$-module $D_{S}$ over $\boldsymbol{B}_{\mathrm{rig}, K}^{\dagger} \hat{\otimes}_{\mathbb{Q}_{p}} S$, we define the Herr complex $C_{\dot{\varphi}, \gamma_{K}}^{\bullet}\left(D_{S}\right)$ of $D_{S}$ concentrated in degree $[0,2]$ as

$$
C_{\varphi, \gamma_{K}}^{\bullet}\left(D_{S}\right)=\left[D_{S}^{\Delta_{K}} \stackrel{d_{1}}{\longrightarrow} D_{S}^{\Delta_{K}} \oplus D_{S}^{\Delta_{K}} \stackrel{d_{2}}{\longrightarrow} D_{S}^{\Delta_{K}}\right],
$$

with $d_{1}(x)=\left(\left(\gamma_{K}-1\right) x,(\varphi-1) x\right)$ and $d_{2}(x, y)=(\varphi-1) x-\left(\gamma_{K}-1\right) y$. One shows that this complex is independent of the choice of $\gamma_{K}$ up to canonical $S$ linear isomorphisms: the isomorphism $C_{\dot{\varphi}, \gamma_{K}}^{\bullet}\left(D_{S}\right) \stackrel{\sim}{\longrightarrow} C_{\varphi, \gamma_{K}^{\prime}}^{\bullet}\left(D_{S}\right)$ is given by $\left[1,1 \oplus\left(\gamma_{K}^{\prime}-1\right) /\left(\gamma_{K}-1\right),\left(\gamma_{K}^{\prime}-1\right) /\left(\gamma_{K}-1\right)\right]$. We will denote the cohomology of $C_{\dot{\varphi}, \gamma_{K}}\left(D_{S}\right)$ by $H^{\bullet}\left(D_{S}\right)$.

By the main result of [Kedlaya et al. 2014], one knows that $H^{i}\left(D_{S}\right)$ is a finitely generated $S$-module. It therefore follows that $H^{i}\left(D_{S}\right)$ commutes with flat base change in $S$. That is, if $S \rightarrow S^{\prime}$ is flat, then $H^{i}\left(D_{S}\right) \otimes_{S} S^{\prime} \cong H^{i}\left(D_{S^{\prime}}\right)$. This enables a cohomology theory for families of $(\varphi, \Gamma)$-modules over general rigid analytic spaces.

Definition 2.2. Let $X$ be a rigid analytic space over $\mathbb{Q}_{p}$, and let $D_{X}$ be a family of $(\varphi, \Gamma)$-modules over $X$. We define $H^{\bullet}\left(D_{X}\right)$ to be the cohomology of the complex of sheaves

$$
C_{\varphi, \gamma_{K}}^{\bullet}\left(D_{X}\right)=\left[D_{X}^{\Delta_{K}} \stackrel{d_{1}}{\longrightarrow} D_{X}^{\Delta_{K}} \oplus D_{X}^{\Delta_{K}} \stackrel{d_{2}}{\longrightarrow} D_{X}^{\Delta_{K}}\right]
$$

in the category of presheaves over $X$, with $d_{1}(x)=\left(\left(\gamma_{K}-1\right) x,(\varphi-1) x\right)$ and $d_{2}(x, y)=(\varphi-1) x-\left(\gamma_{K}-1\right) y$. For each affinoid subdomain $M(S)$ of $X$ and $0 \leq i \leq 2$, the module of sections of $H^{i}\left(D_{X}\right)$ on $M(S)$ is canonically isomorphic to $H^{i}\left(D_{S}\right)$. Hence $H^{i}\left(D_{X}\right)$ forms a coherent $0_{X}$-module by the flat base change property of $H^{i}\left(D_{S}\right)$. 
As a consequence of finiteness of the cohomology of families of $(\varphi, \Gamma)$-modules, by a standard argument we see that, locally on $X$, the complex $C_{\varphi, \gamma_{K}}^{\bullet}\left(D_{X}\right)$ is quasiisomorphic to a complex of locally free coherent sheaves concentrated in degree $[0,2]$. This enables us to flatten the cohomology of families of $(\varphi, \Gamma)$-modules by blowing up the base $X$. The following lemma is a rearrangement of some arguments in [Kedlaya et al. 2014, §6.3]:

Lemma 2.3. Let $X$ be a reduced and irreducible rigid analytic space over $F$, and let $D_{X}$ be a family of $(\varphi, \Gamma)$-modules of rank $d$ over $X$. Then the following statements are true:

(1) There exists a proper birational morphism $\pi: X^{\prime} \rightarrow X$ of reduced rigid analytic spaces over $F$ so that $H^{0}\left(D_{X^{\prime}}\right)$ is flat and $H^{i}\left(D_{X^{\prime}}\right)$ has Tor-dimension $\leq 1$ for each $i=1,2$.

(2) Suppose that $D_{X}^{\prime}$ is a family of $(\varphi, \Gamma)$-modules over $X$ of rank $d^{\prime}$, and that $\lambda: D_{X}^{\prime} \rightarrow D_{X}$ is a morphism between them so that for any $x \in X$ the image of $\lambda_{x}$ is $a(\varphi, \Gamma)$-submodule of rank $d$ of $D_{x}$. Then there exists a proper birational morphism $\pi: X^{\prime} \rightarrow X$ of reduced rigid analytic spaces over $F$ so that the cokernel of $\pi^{*} \lambda$ has Tor-dimension $\leq 1$.

Proof. The upshot is that for a bounded complex $\left(C^{\bullet}, d^{\bullet}\right)$ of locally free coherent sheaves on $X$, there exists a blow-up $\pi: X^{\prime} \rightarrow X$, which depends only on the quasiisomorphism class of $\left(C^{\bullet}, d^{\bullet}\right)$, so that $\pi^{*} d^{i}$ has flat image for each $i$. Furthermore, the construction of $X^{\prime}$ commutes with dominant base change in $X$ (see [Kedlaya et al. 2014, Corollary 6.3.6] for more details). Thus for (1), we can construct $X^{\prime}$ locally and then glue. For (2), let $Q_{X}$ denote the cokernel of $\lambda$. For any $x \in X$, since the image of $\lambda_{x}$ is a $(\varphi, \Gamma)$-submodule of rank $d$, by [Liu 2014, Lemma 5.3.1], we get that $Q_{x}$ is killed by a power of $t$. Now let $M(S)$ be an affinoid subdomain of $X$, and suppose that $D_{S}^{S}$ and $D_{S}^{\prime s}$ are defined for some suitable $s>0$. For $r>s$, set $Q_{S}^{[s, r]}$ to be $D_{S}^{[s, r]} / \lambda\left(D_{S}^{\prime[s, r]}\right)$. Since for any $x \in M(S)$ the fiber of $Q_{S}^{[s, r]}$ at $x$ is killed by a power of $t$, we get that $Q_{S}^{[s, r]}$ is killed by $t^{k}$ for some $k>0$. This yields that $Q_{S}^{[s, r]}$ is a finite $S$-module. Now we apply [Kedlaya et al. 2014, Corollary 6.3.6] to a finite presentation of $Q_{S}^{[s, p s]}$ to get a blow-up $Y$ of $M(S)$ so that the pullback of $Q_{S}^{[s, p s]}$ has Tor-dimension $\leq 1$. Using the fact that $\left(\varphi^{n}\right)^{*} Q_{S}^{[s, p s]} \cong Q_{S}^{\left[p^{n} s, p^{n+1} s\right]}$, we see that $Y$ is also the blow-up obtained by applying [Kedlaya et al. 2014, Corollary 6.3.6] to a finite presentation of $Q_{S}^{\left[s, p^{n+1} s\right]}$ for any positive integer $n$. It therefore follows that for any $r>s$ the pullback of $Q_{S}^{[S, r]}$ has Tor-dimension $\leq 1$; hence the pullback of $Q_{S}$ has Tor-dimension $\leq 1$. Furthermore, the blow-ups for all affinoid subdomains $M(S)$ glue to form a blow-up $X^{\prime}$ of $X$ which satisfies the desired condition. 
Lemma 2.4. Let $X$ be a reduced and irreducible rigid analytic space over $F$. Let $D_{X}^{\prime}$ and $D_{X}$ be families of $(\varphi, \Gamma)$-modules over $X$ of ranks $d^{\prime}$ and $d$ respectively, and let $\lambda: D_{X}^{\prime} \rightarrow D_{X}$ be a morphism between them. Suppose that for any $x \in X$ the image of $\lambda_{x}$ is a $(\varphi, \Gamma)$-submodule of rank $d$ of $D_{x}$. Then there exists a proper birational morphism $\pi: X^{\prime} \rightarrow X$ of reduced rigid analytic spaces over $F$ such that the kernel of $\pi^{*} \lambda$ is a family of $(\varphi, \Gamma)$-modules of rank $d^{\prime}-d$ over $X^{\prime}$, and there exists a Zariski-open dense subset $U \subset X^{\prime}$ such that $\left(\operatorname{ker}\left(\pi^{*} \lambda\right)\right)_{x}=\operatorname{ker}\left(\left(\pi^{*} \lambda\right)_{x}\right)$ for any $x \in U$.

Proof. Let $Q_{X}$ be the cokernel of $\lambda$. By Lemma 2.3, we may suppose that $Q_{X}$ has Tor-dimension $\leq 1$ after adapting $X$. Now let $P_{X}$ denote the kernel of $\lambda$. For any $x \in X$, the Tor spectral sequence computing the cohomology of the complex $\left[D_{X} \underset{\lambda}{\longrightarrow} D_{X}^{\prime}\right] \otimes_{O_{X}}^{L} k(x)$ gives rise to a short exact sequence

$$
0 \longrightarrow P_{x} \longrightarrow \operatorname{ker}\left(\lambda_{x}\right) \longrightarrow \operatorname{Tor}_{1}\left(Q_{X}, k(x)\right) \longrightarrow 0 .
$$

Since the image of $\lambda_{x}$ is a $(\varphi, \Gamma)$-module of rank $d, \operatorname{ker}\left(\lambda_{x}\right)$ is a $(\varphi, \Gamma)$-module of rank $d^{\prime}-d$. Since $Q_{X}$ is killed by a power of $t$ locally on $X$, we get that the last term of the exact sequence is killed by a power of $t$. This yields that $P_{X}$ is a $(\varphi, \Gamma)$ module of rank $d^{\prime}-d$. We therefore conclude that $P_{X}$ is a family of $(\varphi, \Gamma)$-modules of rank $d^{\prime}-d$ over $X$ by [Kedlaya et al. 2014, Corollary 2.1.9]. Furthermore, since $Q_{X}$ has Tor-dimension $\leq 1$, by [Kedlaya et al. 2014, Lemma 6.3.7] we get that the set of $x \in X$ for which $\operatorname{Tor}_{1}\left(Q_{X}, k(x)\right) \neq 0$ forms a nowhere-dense Zariski-closed subset of $X$; this yields the rest of the lemma.

The following proposition modifies part of [Kedlaya et al. 2014, Theorem 6.3.9]:

Proposition 2.5. Let $X$ be a reduced and irreducible rigid analytic space over $F$. Let $D_{X}$ be a family of $(\varphi, \Gamma)$-modules of rank $d$ over $X$, and let $\delta: K^{\times} \rightarrow \mathcal{O}(X)^{\times}$ be a continuous character. Suppose that there exists a Zariski-dense subset $Z$ of closed points of $X$ and a positive integer $c \leq d$ such that, for every $z \in Z$, $H^{0}\left(D_{z}^{\vee}\left(\delta_{z}\right)\right)$ is a c-dimensional $k(z)$-vector space. Then there exists a proper birational morphism $\pi: X^{\prime} \rightarrow X$ of reduced rigid analytic spaces over $F$ and a morphism $\lambda: D_{X^{\prime}} \rightarrow M_{X^{\prime}}=\left(\boldsymbol{B}_{\mathrm{rig}, K}^{\dagger} \hat{\otimes}_{\mathbb{Q}_{p}} \mathcal{O}_{X^{\prime}}\right)(\delta) \otimes_{\mathbb{O}_{X^{\prime}}}$ L of $(\varphi, \Gamma)$-modules,

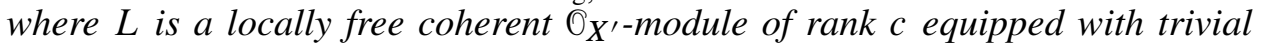
$(\varphi, \Gamma)$-actions, such that:

(1) For any $x \in X^{\prime}$, the image of $\lambda_{x}$ is a $(\varphi, \Gamma)$-submodule of rank $c$.

(2) The kernel of $\lambda$ is a family of $(\varphi, \Gamma)$-modules of rank $d-c$ over $X^{\prime}$, and there exists a Zariski-open dense subset $U \subset X^{\prime}$ such that $(\operatorname{ker} \lambda)_{x}=\operatorname{ker}\left(\lambda_{x}\right)$ for any $x \in U$. 
Proof. Using Lemma 2.3, we first choose a proper birational morphism $\pi: X^{\prime} \rightarrow X$ with $X^{\prime}$ reduced such that $N_{X^{\prime}}=\pi^{*}\left(D_{X}^{\vee}(\delta)\right)$ satisfies the conditions that $H^{0}\left(N_{X^{\prime}}\right)$ is flat and $H^{i}\left(N_{X^{\prime}}\right)$ has Tor-dimension $\leq 1$ for each $i=1,2$. Then, for any $x \in X^{\prime}$, the base change spectral sequence $E_{2}^{i, j}=\operatorname{Tor}_{-i}\left(H^{j}\left(N_{X^{\prime}}\right), k(x)\right) \Rightarrow H^{i+j}\left(N_{x}\right)$ gives a short exact sequence (using that $H^{1}\left(N_{X^{\prime}}\right)$ has Tor-dimension $\leq 1$ and $N_{X^{\prime}}$ is flat)

$$
0 \longrightarrow H^{0}\left(N_{X^{\prime}}\right) \otimes_{0_{X^{\prime}}} k(x) \longrightarrow H^{0}\left(N_{x}\right) \longrightarrow \operatorname{Tor}_{1}\left(H^{1}\left(N_{X^{\prime}}\right), k(x)\right) \longrightarrow 0 .
$$

Since $H^{1}\left(N_{X^{\prime}}\right)$ has Tor-dimension $\leq 1$, by [Kedlaya et al. 2014, Lemma 6.3.7] the set of $x \in X^{\prime}$ for which the last term of the above exact sequence does not vanish forms a nowhere-dense Zariski-closed subset $V$. For any $z \in \pi^{-1}(Z) \backslash V$, we deduce that $H^{0}\left(N_{X^{\prime}}\right) \otimes_{\mathcal{O}_{X^{\prime}}} k(z)$ is a $c$-dimensional $k(z)$-vector space. Since $H^{0}\left(N_{X^{\prime}}\right)$ is flat and $\pi^{-1}(Z) \backslash V$ is a Zariski-dense subset of $X^{\prime}$, we get that $H^{0}\left(N_{X^{\prime}}\right)$ is locally free of constant rank $c$. Let $L$ be its dual coherent $0_{X^{\prime}}$-module. Then the natural map $\left(\boldsymbol{B}_{\mathrm{rig}, K}^{\dagger} \hat{\otimes}_{\mathbb{Q}_{p}} \mathcal{O}_{X^{\prime}}\right) H^{0}\left(N_{X^{\prime}}\right) \rightarrow N_{X^{\prime}}$ gives rise to a map

$$
\lambda: D_{X^{\prime}} \rightarrow M_{X^{\prime}}=\left(\boldsymbol{B}_{\mathrm{rig}, K}^{\dagger} \widehat{\otimes}_{\mathbb{Q}_{p}} \mathcal{O}_{X^{\prime}}\right)(\delta) \otimes_{\mathcal{O}_{X^{\prime}}} L .
$$

For any $x \in X^{\prime}$, since the map $H^{0}\left(N_{X^{\prime}}\right) \otimes_{\bigcirc_{X^{\prime}}} k(x) \rightarrow H^{0}\left(N_{x}\right)$ is injective, we get that the image of $\lambda_{x}$ is a rank-c $(\varphi, \Gamma)$-submodule of $M_{x}$. We thus conclude the proposition using Lemma 2.4.

\section{Families of Hodge-Tate $(\varphi, \Gamma)$-modules}

From now on, let $S$ be an affinoid algebra over $F$. Recall that for any $n \geq n(s)$ there is a continuous $\Gamma$-equivariant injective map

$$
\iota_{n}: \boldsymbol{B}_{\mathrm{rig}, K}^{\dagger, s} \rightarrow K_{n} \llbracket t \rrbracket .
$$

It is defined as the composite

$$
\boldsymbol{B}_{K}^{\dagger, s} \subset \widetilde{\boldsymbol{B}}^{\dagger, s} \stackrel{\varphi^{-n}}{\longrightarrow} \widetilde{\boldsymbol{B}}^{\dagger, p^{-n} s} \subset \widetilde{\boldsymbol{B}}^{+} \subset \boldsymbol{B}_{\mathrm{dR}}^{+},
$$

and it factors through $K_{n} \llbracket t \rrbracket$ (see [Berger 2002, §2] for more details about $\iota_{n}$ ). In particular, we have $\iota_{n+1} \circ \varphi=\iota_{n}$. The map $\iota_{n}$ induces a continuous $\Gamma$-equivariant map

$$
\iota_{n}: \boldsymbol{B}_{\mathrm{rig}, K}^{\dagger, s} \hat{\otimes}_{\mathbb{Q}_{p}} S \rightarrow K_{n} \llbracket t \rrbracket \hat{\otimes}_{\mathbb{Q}_{p}} S .
$$

Definition 3.1. Let $D_{S}$ be a $(\varphi, \Gamma)$-module of rank $d$ over $\boldsymbol{B}_{\text {rig, } K}^{\dagger} \widehat{\otimes}_{\mathbb{Q}_{p}} S$. For any positive integer $n$, if $D_{S}^{r_{n}}$ is defined, then for any $0<s \leq r_{n}$ we set

$$
\mathrm{D}_{\text {dif }}^{+, K_{n}}\left(D_{S}\right)=D_{S}^{S} \otimes_{B_{\mathrm{rig}, K}^{\dagger, s} \hat{\otimes}_{\mathbb{Q}_{p}} S, \iota_{n}}\left(K_{n} \llbracket t \rrbracket \hat{\otimes}_{\mathbb{Q}_{p}} S\right)
$$

and

$$
\mathrm{D}_{\text {dif }}^{K_{n}}\left(D_{S}\right)=\mathrm{D}_{\text {dif }}^{+, K_{n}}\left(D_{S}\right)[1 / t]
$$


We also denote the natural map

$$
D_{S}^{S} \rightarrow \mathrm{D}_{\mathrm{dif}}^{+, K_{n}}\left(D_{S}\right)
$$

by $\iota_{n}$, and call it the localization map. Define $\mathrm{D}_{\operatorname{Sen}}^{K_{n}}\left(D_{S}\right)=\mathrm{D}_{\mathrm{dif}}^{+, K_{n}}\left(D_{S}\right) /(t)$. For $D_{S}=\mathrm{D}_{\text {rig }}^{\dagger}\left(V_{S}\right)$ coming from a finite locally free $S$-linear representation $V_{S}$, we write $\mathrm{D}_{\text {dif }}^{+, K_{n}}\left(V_{S}\right)$ and $\mathrm{D}_{\text {Sen }}^{K_{n}}\left(V_{S}\right)$ for $\mathrm{D}_{\text {dif }}^{+, K_{n}}\left(D_{S}\right)$ and $\mathrm{D}_{\text {Sen }}^{K_{n}}\left(D_{S}\right)$ respectively. When the base field is clear from the context, we write $\mathrm{D}_{\text {dif }}^{+, n}\left(D_{S}\right)$ and $\mathrm{D}_{\text {Sen }}^{n}\left(D_{S}\right)$ instead of $\mathrm{D}_{\text {dif }}^{+, K_{n}}\left(D_{S}\right)$ and $\mathrm{D}_{\mathrm{Sen}}^{K_{n}}\left(D_{S}\right)$ for simplicity.

Definition 3.2. We call $D_{S}$ Hodge-Tate with Hodge-Tate weights in $[a, b]$ if there exists a positive integer $n$ such that the natural map

$$
\left(\bigoplus_{a \leq i \leq b} \mathrm{D}_{\mathrm{Sen}}^{n}\left(D_{S}(-i)\right)\right)^{\Gamma} \otimes_{K \otimes_{\mathbb{Q} p} S}\left(K_{n} \otimes_{\mathbb{Q}_{p}} S\right)\left[t, t^{-1}\right] \longrightarrow \bigoplus_{i \in \mathbb{Z}} \mathrm{D}_{\mathrm{Sen}}^{n}\left(D_{S}(-i)\right)
$$

is an isomorphism. We denote by $h_{\mathrm{HT}}\left(D_{S}\right)$ the smallest $n$ which satisfies this condition, and we define $D_{\mathrm{HT}}\left(D_{S}\right)=\left(\bigoplus_{a \leq i \leq b} \mathrm{D}_{\mathrm{Sen}}^{h_{\mathrm{HT}}\left(D_{S}\right)}\left(D_{S}(-i)\right)\right)^{\Gamma}$.

Lemma 3.3. Let $D_{S}$ be a Hodge-Tate $(\varphi, \Gamma)$-module over $\boldsymbol{B}_{\mathrm{rig}, K}^{\dagger} \hat{\otimes}_{\mathbb{Q}_{p}} S$ with weights in $[a, b]$. Then for any $n \geq h_{\mathrm{HT}}\left(D_{S}\right)$ (3.2.1) is an isomorphism and $\mathrm{D}_{\mathrm{Sen}}^{n}\left(D_{S}(-i)\right)^{\Gamma}=\mathrm{D}_{\mathrm{Sen}}^{h_{\mathrm{HT}}\left(D_{S}\right)}\left(D_{S}(-i)\right)^{\Gamma}$ for any $i \in[a, b]$. As a consequence, we have $\left(\bigoplus_{a \leq i \leq b} \mathrm{D}_{\text {Sen }}^{n}\left(D_{S}(-i)\right)\right)^{\Gamma}=D_{\mathrm{HT}}\left(D_{S}\right)$.

Proof. Tensoring with $K_{n} \otimes_{\mathbb{Q}_{p}} S[t, 1 / t]$ on both sides of the map

$$
\begin{aligned}
\left(\bigoplus_{a \leq i \leq b} \mathrm{D}_{\mathrm{Sen}}^{h_{\mathrm{HT}}\left(D_{S}\right)}\left(D_{S}(-i)\right)\right)^{\Gamma} \otimes_{K \otimes_{\mathbb{Q} p} S}\left(K_{h_{\mathrm{HT}}\left(D_{S}\right)} \otimes_{\mathbb{Q}_{p}} S\right)\left[t, t^{-1}\right] & \\
& \longrightarrow \bigoplus_{i \in \mathbb{Z}} \mathrm{D}_{\mathrm{Sen}}^{h_{\mathrm{HT}}\left(D_{S}\right)}\left(D_{S}(-i)\right),
\end{aligned}
$$

we get that the natural map

$$
\begin{aligned}
&\left(\bigoplus_{a \leq i \leq b} \mathrm{D}_{\mathrm{Sen}}^{h_{\mathrm{HT}}\left(D_{S}\right)}\left(D_{S}(-i)\right)\right)^{\Gamma} \otimes_{K \otimes_{\mathbb{Q} p} S}\left(K_{n} \otimes_{\mathbb{Q}_{p}} S\right)\left[t, t^{-1}\right] \\
& \longrightarrow \bigoplus_{i \in \mathbb{Z}} \mathrm{D}_{\mathrm{Sen}}^{n}\left(D_{S}(-i)\right)
\end{aligned}
$$

is an isomorphism. Taking $\Gamma$-invariants on both sides, we get

$$
\left(\bigoplus_{a \leq i \leq b} \mathrm{D}_{\mathrm{Sen}}^{h_{\mathrm{HT}}\left(D_{S}\right)}\left(D_{S}(-i)\right)\right)^{\Gamma}=\left(\bigoplus_{a \leq i \leq b} \mathrm{D}_{\mathrm{Sen}}^{n}\left(D_{S}(-i)\right)\right)^{\Gamma}
$$

Remark 3.4. If $D_{S}$ is Hodge-Tate with weights in $[a, b]$, then, by taking $\Gamma$ invariants on both sides of (3.2.1), we see that $\mathrm{D}_{\text {Sen }}^{n}\left(D_{S}(-i)\right)^{\Gamma}=0$ for any $n \geq h_{\mathrm{HT}}\left(D_{S}\right)$ and $i \notin[a, b]$. 
Lemma 3.5. If $D_{S}$ is a Hodge-Tate $(\varphi, \Gamma)$-module over $\boldsymbol{B}_{\mathrm{rig}, K}^{\dagger} \hat{\otimes}_{\mathbb{Q}_{p}} S$ with weights in $[a, b]$, then for any morphism $S \rightarrow R$ of affinoid algebras over $K, D_{R}$ is HodgeTate with weights in $[a, b]$ and $h_{\mathrm{HT}}\left(D_{R}\right) \leq h_{\mathrm{HT}}\left(D_{S}\right)$. Furthermore, the natural map

$$
\mathrm{D}_{\text {Sen }}^{n}\left(D_{S}(i)\right)^{\Gamma} \otimes_{S} R \rightarrow \mathrm{D}_{\text {Sen }}^{n}\left(D_{R}(i)\right)^{\Gamma}
$$

is an isomorphism for any $i \in \mathbb{Z}$ and $n \geq h_{\mathrm{HT}}\left(D_{S}\right)$. As a consequence, the natural map $D_{\mathrm{HT}}\left(D_{S}\right) \otimes_{S} R \rightarrow D_{\mathrm{HT}}\left(D_{R}\right)$ is an isomorphism.

Proof. Let $n \geq h_{\mathrm{HT}}\left(D_{S}\right)$. Tensoring with $R$ over $S$ on both sides of (3.2.1), we get that the natural map

$$
\begin{aligned}
\left(\bigoplus_{a \leq i \leq b} \mathrm{D}_{\mathrm{Sen}}^{n}\left(D_{S}(-i)\right)^{\Gamma} \otimes_{S} R\right) \otimes_{K \otimes_{\mathbb{Q}_{p}} R}\left(K_{n} \otimes_{\mathbb{Q}_{p}} R\right)\left[t, t^{-1}\right] & \\
& \longrightarrow \bigoplus_{i \in \mathbb{Z}} \mathrm{D}_{\mathrm{Sen}}^{n}\left(D_{R}(-i)\right)
\end{aligned}
$$

is an isomorphism. Comparing $\Gamma$-invariants on both sides, we get that the natural map

$$
\mathrm{D}_{\text {Sen }}^{n}\left(D_{S}(-i)\right)^{\Gamma} \otimes_{S} R \rightarrow \mathrm{D}_{\text {Sen }}^{n}\left(D_{R}(-i)\right)^{\Gamma}
$$

is an isomorphism for any $a \leq i \leq b$. This implies that the natural map

$$
\left(\bigoplus_{a \leq i \leq b} \mathrm{D}_{\mathrm{Sen}}^{n}\left(D_{R}(-i)\right)^{\Gamma}\right) \otimes_{K \otimes_{\mathbb{Q}_{p}} R}\left(K_{n} \otimes_{\mathbb{Q}_{p}} R\right)\left[t, t^{-1}\right] \longrightarrow \bigoplus_{i \in \mathbb{Z}} \mathrm{D}_{\mathrm{Sen}}^{n}\left(D_{R}(-i)\right) \text {. }
$$

is an isomorphism.

Corollary 3.6. If $D_{S}$ is a Hodge-Tate $(\varphi, \Gamma)$-module of rank d over $\boldsymbol{B}_{\text {rig, } K}^{\dagger} \widehat{\otimes}_{\mathbb{Q}_{p}} S$, then $D_{\mathrm{HT}}\left(D_{S}\right)$ is a locally free coherent $K \otimes_{\mathbb{Q}_{p}} S$-module of rank $d$.

Proof. By the previous lemma, it suffices to treat the case that $S$ is a finite extension of $K$; this is clear from the isomorphism (3.2.1).

Definition 3.7. Let $X$ be a rigid analytic space over $F$, and let $D_{X}$ be a family of $(\varphi, \Gamma)$-modules of rank $d$ over $X$. We call $D_{X}$ Hodge-Tate with weights in $[a, b]$ if for some (hence any) admissible cover $\left\{M\left(S_{i}\right)\right\}_{i \in I}$ of $X, D_{S_{i}}$ is Hodge-Tate with weights in $[a, b]$ for any $i \in I$. We define $D_{\mathrm{HT}}\left(D_{X}\right)$ to be the gluing of all the $D_{\mathrm{HT}}\left(D_{S_{i}}\right)$.

Lemma 3.8. Let $D_{S}$ be a $(\varphi, \Gamma)$-module over $\boldsymbol{B}_{\mathrm{rig}, K}^{\dagger} \hat{\otimes}_{\mathbb{Q}_{p}} S$. Then (3.2.1) is an isomorphism if and only if the natural map

$$
\bigoplus_{a \leq i \leq b} \mathrm{D}_{\text {Sen }}^{n}\left(D_{S}\right)^{\Gamma_{n}=\chi^{i}} \longrightarrow \mathrm{D}_{\text {Sen }}^{n}\left(D_{S}\right)
$$

is an isomorphism. Furthermore, if this is the case, then (3.2.1) holds for $n$. 
Proof. For the " $\Rightarrow$ " part, since (3.2.1) is an isomorphism, we deduce that

$$
\mathrm{D}_{\text {Sen }}^{n}\left(D_{S}\right)=\bigoplus_{a \leq i \leq b} t^{i} \cdot \mathrm{D}_{\text {Sen }}^{n}\left(D_{S}(-i)\right)^{\Gamma} \otimes_{K \otimes_{\mathbb{Q}_{p}} S}\left(K_{n} \otimes_{\mathbb{Q}_{p}} S\right) .
$$

Note that $t^{i} \cdot \mathrm{D}_{\mathrm{Sen}}^{n}\left(D_{S}(-i)\right)^{\Gamma} \subseteq \mathrm{D}_{\mathrm{Sen}}^{n}\left(D_{S}\right)^{\Gamma_{n}=\chi^{i}}$. Hence (3.8.2) implies that (3.8.1) is surjective. On the other hand, it is clear that (3.2.1) is injective; hence it is an isomorphism. Conversely, suppose that (3.8.1) is an isomorphism. Note that

$$
\begin{aligned}
\mathrm{D}_{\mathrm{Sen}}^{n}\left(D_{S}\right)^{\Gamma_{n}=\chi^{i}} & =t^{i} \cdot \mathrm{D}_{\mathrm{Sen}}^{n}\left(D_{S}(-i)\right)^{\Gamma_{n}} \\
& =\left(t^{i} \cdot \mathrm{D}_{\mathrm{Sen}}^{n}\left(D_{S}(-i)\right)^{\Gamma}\right) \otimes_{K \otimes_{\mathbb{Q}_{p}} S}\left(K_{n} \otimes_{\mathbb{Q}_{p}} S\right),
\end{aligned}
$$

where the latter equality follows from [Berger and Colmez 2008, Proposition 2.2.1]. This implies that $D_{S}$ satisfies (3.8.2), yielding that $D_{S}$ satisfies (3.2.1).

Proposition 3.9. Let $S$ be reduced, and let $D_{S}$ be a $(\varphi, \Gamma)$-module over $\boldsymbol{B}_{\mathrm{rig}, K}^{\dagger} \hat{\otimes} S$. Suppose that there exists a Zariski-dense subset $Z \subset M(S)$ such that $D_{z}$ is HodgeTate with weights in $[a, b]$ for any $z \in Z$ and $\sup _{z \in Z}\left\{h_{\mathrm{HT}}\left(D_{z}\right)\right\}<\infty$. Then $D_{S}$ is Hodge-Tate with weights in $[a, b]$.

Proof. Let $n \geq \sup _{z \in Z}\left\{h_{\mathrm{HT}}\left(D_{z}\right)\right\}$ such that $D_{S}^{n}$ is defined, and let $\gamma$ be a topological generator of $\Gamma_{n}$. For any $a \leq i \leq b$, let $p_{i}$ denote the operator

$$
\prod_{a \leq j \leq b, j \neq i} \frac{\gamma-\chi^{j}(\gamma)}{\chi^{i}(\gamma)-\chi^{j}(\gamma)},
$$

and let $M_{i}=p_{i}\left(\mathrm{D}_{\mathrm{Sen}_{i}}^{n}\left(D_{S}\right)\right)$. It is clear that $p_{i}$ is the identity on $\mathrm{D}_{\mathrm{Sen}}^{n}\left(D_{S}\right)^{\Gamma_{n}=\chi^{i}}$; hence $\mathrm{D}_{\mathrm{Sen}}^{n}\left(D_{S}\right)^{\Gamma_{n}=\chi^{i}} \subseteq M_{i}$. On the other hand, for any $z \in Z$, since $D_{z}$ is HodgeTate with weights in $[a, b]$ and $h_{\mathrm{HT}}\left(D_{z}\right) \leq n$, we deduce from Lemma 3.8 that $p_{i}\left(\mathrm{D}_{\text {Sen }}^{n}\left(D_{z}\right)\right)=\mathrm{D}_{\text {Sen }}^{n}\left(D_{z}\right)^{\Gamma_{n}=\chi^{i}}$. This implies that $M_{i}$ maps onto $\mathrm{D}_{\text {Sen }}^{n}\left(D_{z}\right)^{\Gamma_{n}=\chi^{i}}$ under the specialization $\mathrm{D}_{\text {Sen }}^{n}\left(D_{S}\right) \rightarrow \mathrm{D}_{\text {Sen }}^{n}\left(D_{z}\right)$. Since $S$ is reduced and $Z$ is Zariski-dense, we obtain $M_{i} \subseteq \mathrm{D}_{\text {Sen }}^{n}(D)^{\Gamma_{n}=\chi^{i}}$; hence $M_{i}=\mathrm{D}_{\text {Sen }}^{n}(D)^{\Gamma_{n}=\chi^{i}}$. Let $M=\bigoplus_{a \leq i \leq b} M_{i}$. We claim that the natural inclusion $M \subseteq \mathrm{D}_{\text {Sen }}^{n}\left(D_{S}\right)$ is an isomorphism. In fact, for any $z \in Z$, since $\mathrm{D}_{\text {Sen }}^{n}\left(D_{z}\right)=\bigoplus_{a \leq i \leq b} \mathrm{D}_{\text {Sen }}^{n}\left(D_{z}\right)^{\Gamma_{n}=\chi^{i}}$, we have that $M$ maps onto $\mathrm{D}_{\mathrm{Sen}}^{n}\left(D_{z}\right)$. Thus $\mathrm{D}_{\text {Sen }}^{n}\left(D_{S}\right) / M$ vanishes at $z$. We therefore conclude that $\mathrm{D}_{\text {Sen }}^{n}\left(D_{S}\right) / M=0$ because $S$ is reduced and $Z$ is Zariskidense. By Lemma 3.8 and the claim, we conclude that $D_{S}$ is Hodge-Tate with weights in $[a, b]$.

\section{Families of de $\operatorname{Rham}(\varphi, \Gamma)$-modules}

Definition 4.1. Let $D_{S}$ be a $(\varphi, \Gamma)$-module of rank $d$ over $\boldsymbol{B}_{\text {rig, } K}^{\dagger} \hat{\otimes}_{\mathbb{Q}_{p}} S$. For any positive integer $n$, if $D_{S}^{r_{n}}$ is defined, then we equip $\mathrm{D}_{\text {dif }}^{n}\left(D_{S}\right)$ with the filtration 
Fil $^{i} \mathrm{D}_{\text {dif }}^{n}\left(D_{S}\right)=t^{i} \mathrm{D}_{\text {dif }}^{+, n}\left(D_{S}\right)$. We call $D_{S}$ de Rham with weights in $[a, b]$ if there exists a positive integer $n$ such that:

(1) The natural map

$$
\mathrm{D}_{\text {dif }}^{n}\left(D_{S}\right)^{\Gamma} \otimes_{K \otimes_{\mathbb{Q}_{p}} S}\left(K_{n} \otimes_{\mathbb{Q}_{p}} S\right) \llbracket t \rrbracket[1 / t] \longrightarrow \mathrm{D}_{\text {dif }}^{n}\left(D_{S}\right)
$$

is an isomorphism.

(2) $\mathrm{Fil}^{-b}\left(\mathrm{D}_{\mathrm{dif}}^{n}\left(D_{S}\right)^{\Gamma}\right)=D_{S}$ and Fil ${ }^{-a+1}\left(\mathrm{D}_{\mathrm{dif}}^{n}\left(D_{S}\right)^{\Gamma}\right)=0$, where Fil ${ }^{i}\left(\mathrm{D}_{\mathrm{dif}}^{n}\left(D_{S}\right)^{\Gamma}\right)$ is the induced filtration on $\mathrm{D}_{\text {dif }}^{n}\left(D_{S}\right)^{\Gamma}$.

We denote the smallest $n$ satisfying these conditions by $h_{\mathrm{dR}}\left(D_{S}\right)$, and we set $D_{\mathrm{dR}}\left(D_{S}\right)=\mathrm{D}_{\mathrm{dif}}^{h_{\mathrm{dR}}\left(D_{S}\right)}\left(D_{S}\right)^{\Gamma}$.

Lemma 4.2. Let $D$ be a de Rham $(\varphi, \Gamma)$-module over $\boldsymbol{B}_{\mathrm{rig}, K}^{\dagger} \hat{\otimes}_{\mathbb{Q}_{p}} S$. Then, for any $n \geq h_{\mathrm{dR}}\left(D_{S}\right), \mathrm{D}_{\mathrm{dif}}^{n}\left(D_{S}\right)^{\Gamma}=D_{\mathrm{dR}}\left(D_{S}\right)$.

Proof. We tensor with $K_{n+1} \otimes_{\mathbb{Q}_{p}} S \llbracket t \rrbracket[1 / t]$ on both sides of the map

$$
\mathrm{D}_{\text {dif }}^{h_{\mathrm{dR}}\left(D_{S}\right)}\left(D_{S}\right)^{\Gamma} \otimes_{K \otimes_{\mathbb{Q}_{p}} S}\left(K_{h_{\mathrm{dR}}\left(D_{S}\right)} \otimes_{\mathbb{Q}_{p}} S\right) \llbracket t \rrbracket[1 / t] \longrightarrow \mathrm{D}_{\mathrm{dif}}^{h_{\mathrm{dR}}\left(D_{S}\right)}\left(D_{S}\right),
$$

yielding that the map

$$
\mathrm{D}_{\mathrm{dif}}^{h_{\mathrm{d}}\left(D_{S}\right)}\left(D_{S}\right)^{\Gamma} \otimes_{K \otimes_{\mathbb{Q}_{p}} S}\left(K_{n} \otimes_{\mathbb{Q}_{p}} S\right) \llbracket t \rrbracket[1 / t] \longrightarrow \mathrm{D}_{\mathrm{dif}}^{n}\left(D_{S}\right)
$$

is an isomorphism. Comparing $\Gamma$-invariants on both sides, we get the desired result.

Lemma 4.3. If $D$ is a de Rham $(\varphi, \Gamma)$-module of rank $d$ over $\boldsymbol{B}_{\mathrm{rig}, K}^{\dagger} \hat{\otimes}_{\mathbb{Q}_{p}} S$ with weights in $[a, b]$, then $D$ is Hodge-Tate with weights in $[a, b]$ and $h_{\mathrm{HT}}\left(D_{S}\right) \leq$ $h_{\mathrm{dR}}\left(D_{S}\right)$. Furthermore, we have $\mathrm{Gr}^{i} D_{\mathrm{dR}}\left(D_{S}\right)=\mathrm{D}_{\mathrm{Sen}}^{n}\left(D_{S}(i)\right)^{\Gamma}$ under the identifcation $\mathrm{Gr}^{i} \mathrm{D}_{\mathrm{dif}}^{n}\left(D_{S}\right)=\mathrm{D}_{\mathrm{Sen}}^{n}\left(D_{S}(i)\right)$ for any $n \geq h_{\mathrm{dR}}\left(D_{S}\right)$.

Proof. Let $n \geq h_{\mathrm{dR}}\left(D_{S}\right)$. Since (4.1.1) is an isomorphism, we deduce that the natural map of graded modules

$$
\bigoplus_{i \in \mathbb{Z}} \mathrm{Gr}^{i} D_{\mathrm{dR}}\left(D_{S}\right) \otimes_{K \otimes_{\mathbb{Q}_{p}} S}\left(K_{n} \otimes_{\mathbb{Q}_{p}} S\right)\left[t, t^{-1}\right] \longrightarrow \bigoplus_{i \in \mathbb{Z}} \mathrm{D}_{\mathrm{Sen}}^{n}\left(D_{S}(i)\right)
$$

is surjective. On the other hand, since $t^{i} \cdot \mathrm{Gr}^{-i} D_{\mathrm{dR}}\left(D_{S}\right) \subset \mathrm{D}_{\text {Sen }}^{n}\left(D_{S}\right)$, we have that the natural map

$$
\bigoplus_{a \leq i \leq b} t^{i} \cdot \mathrm{Gr}^{-i} D_{\mathrm{dR}}\left(D_{S}\right) \longrightarrow \mathrm{D}_{\mathrm{Sen}}^{n}\left(D_{S}\right)
$$

is injective. This implies that (4.3.1) is injective; hence it is an isomorphism. Comparing $\Gamma$-invariants on both sides, we get $\operatorname{Gr}^{i} D_{\mathrm{dR}}\left(D_{S}\right)=\mathrm{D}_{\mathrm{Sen}}^{n}\left(D_{S}(i)\right)^{\Gamma}$ for each $i \in \mathbb{Z}$. 
Lemma 4.4. If $D_{S}$ is a de $\operatorname{Rham}(\varphi, \Gamma)$-module over $\boldsymbol{B}_{\mathrm{rig}, K}^{\dagger} \widehat{\otimes}_{\mathbb{Q}_{p}} S$, then, for any morphism $S \rightarrow R$ of affinoid algebras over $K, D_{R}$ is de Rham with weights in $[a, b]$ and $h_{\mathrm{dR}}\left(D_{R}\right) \leq h_{\mathrm{dR}}\left(D_{S}\right)$. Furthermore, the natural maps Fil ${ }^{i} D_{\mathrm{dR}}\left(D_{S}\right) \otimes_{S} R \rightarrow$ Fil $^{i} D_{\mathrm{dR}}\left(D_{R}\right)$ are isomorphisms for all $i \in \mathbb{Z}$.

Proof. Let $n \geq h_{\mathrm{dR}}\left(D_{S}\right)$. Tensoring with $\left(K_{n} \otimes_{\mathbb{Q}_{p}} R\right) \llbracket t \rrbracket[1 / t]$ on both sides of (4.1.1), we get that the natural map

$$
\left(\mathrm{D}_{\mathrm{dif}}^{n}\left(D_{S}\right)^{\Gamma} \otimes_{S} R\right) \otimes_{K \otimes_{\mathbb{Q} p} R}\left(K_{n} \otimes_{\mathbb{Q}_{p}} R\right) \llbracket t \rrbracket[1 / t] \longrightarrow \mathrm{D}_{\mathrm{dif}}^{n}\left(D_{R}\right) .
$$

is an isomorphism. Comparing $\Gamma$-invariants on both sides of (4.4.1), we get that the natural map $\mathrm{D}_{\text {dif }}^{n}\left(D_{S}\right)^{\Gamma} \otimes_{S} R \rightarrow \mathrm{D}_{\text {dif }}^{n}\left(D_{R}\right)^{\Gamma}$ is an isomorphism; hence $D_{R}$ is de Rham. Then, by Lemmas 3.5 and 4.3, we deduce that the natural map $\operatorname{Gr}^{i}\left(D_{\mathrm{dR}}\left(D_{S}\right)\right) \otimes_{S} R \rightarrow \mathrm{Gr}^{i}\left(D_{\mathrm{dR}}\left(D_{R}\right)\right)$ is an isomorphism. This implies the rest of the lemma.

Corollary 4.5. If $D_{S}$ is a de Rham $(\varphi, \Gamma)$-module of rank $d$ over $\boldsymbol{B}_{\text {rig, } K}^{\dagger} \widehat{\otimes}_{\mathbb{Q}_{p}} S$, then $D_{\mathrm{dR}}\left(D_{S}\right)$ is a locally free coherent $K \otimes_{\mathbb{Q}_{p}} S$-module of rank $d$.

Proof. We first note that, for each $i \in \mathbb{Z}, \operatorname{Gr}^{i}\left(D_{\mathrm{dR}}\left(D_{S}\right)\right)$, which is isomorphic to $\mathrm{D}_{\text {Sen }}^{n}\left(D_{S}(i)\right)^{\Gamma}$ by Lemma 4.3 , is a coherent $K \otimes_{\mathbb{Q}_{p}} S$-module. We then deduce that $D_{\mathrm{dR}}\left(D_{S}\right)$ is a coherent $K \otimes_{\mathbb{Q}_{p}} S$-module. Using Lemma 4.4, it then suffices to treat the case that $S$ is a finite extension of $K$; this follows easily from the isomorphism (4.1.1).

Definition 4.6. Let $X$ be a rigid analytic space over $F$, and let $D_{X}$ be a family of $(\varphi, \Gamma)$-modules of rank $d$ over $X$. We call $D_{X}$ de Rham if for some (hence any) admissible cover $\left\{M\left(S_{i}\right)\right\}_{i \in I}$ of $X, D_{S_{i}}$ is de Rham with weights in $[a, b]$ for any $i \in I$. We define $D_{\mathrm{dR}}\left(D_{X}\right)$ to be the gluing of all the $D_{\mathrm{dR}}\left(D_{S_{i}}\right)$.

Lemma 4.7. If $D_{S}$ is a de Rham $(\varphi, \Gamma)$-module over $\boldsymbol{B}_{\mathrm{rig}, K}^{\dagger} \widehat{\otimes}_{\mathbb{Q}_{p}} S$ of rank $d$ with weights in $[a, b]$, then

$$
t^{-a} \mathrm{D}_{\mathrm{dif}}^{+, n}\left(D_{S}\right) \subset D_{\mathrm{dR}}\left(D_{S}\right) \otimes_{K \otimes_{\mathbb{Q}_{p}} S}\left(K_{n} \otimes_{\mathbb{Q}_{p}} S\right) \llbracket t \rrbracket \subset t^{-b} \mathrm{D}_{\mathrm{dif}}^{+, n}\left(D_{S}\right)
$$

for any $n \geq h_{\mathrm{dR}}\left(D_{S}\right)$.

Proof. Since $\mathrm{Gr}^{-b} D_{\mathrm{dR}}\left(D_{S}\right)=D_{\mathrm{dR}}\left(D_{S}\right)$, we get $D_{\mathrm{dR}}\left(D_{S}\right) \subset t^{-b} \mathrm{D}_{\mathrm{dif}}^{+, n}\left(D_{S}\right)$; hence $D_{\mathrm{dR}}\left(D_{S}\right) \otimes_{K \otimes_{\mathbb{Q} p} S}\left(K_{n} \otimes_{\mathbb{Q}_{p}} S\right) \llbracket t \rrbracket \subset t^{-b} \mathrm{D}_{\mathrm{dif}}^{+, n}\left(D_{S}\right)$. By the proof of Lemma 4.3, we know that the natural map (4.3.1) is an isomorphism of graded modules. By the facts that $\mathrm{Gr}^{i} D_{\mathrm{dR}}\left(D_{S}\right)=0$ for $i \geq-a+1$ and Fil ${ }^{i} \mathrm{D}_{\mathrm{dif}}^{n}\left(D_{S}\right)$ is $t$-adically complete, we thus deduce that $t^{-a} \mathrm{D}_{\text {dif }}^{+, n}\left(D_{S}\right) \subset D_{\mathrm{dR}}\left(D_{S}\right) \otimes_{K \otimes_{\mathbb{Q} p} S}\left(K_{n} \otimes_{\mathbb{Q}_{p}} S\right) \llbracket t \rrbracket$.

Lemma 4.8. Let $D_{S}$ be a Hodge-Tate $(\varphi, \Gamma)$-module over $\boldsymbol{B}_{\text {rig, } K}^{\dagger} \widehat{\otimes}_{\mathbb{Q}_{p}} S$ with weights in $[a, b]$. Then, for any $k \geq b-a+1, i \in[a, b], n \geq h_{\mathrm{HT}}\left(D_{S}\right)$ and $\gamma \in \Gamma_{n}$, the map $\gamma-\chi^{i}(\gamma): t^{k} \mathrm{D}_{\mathrm{dif}}^{+, n}\left(D_{S}\right) \rightarrow t^{k} \mathrm{D}_{\mathrm{dif}}^{+, n}\left(D_{S}\right)$ is bijective. 
Proof. Since $\mathrm{D}_{\text {dif }}^{+, n}\left(D_{S}\right)$ is $t$-adically complete, it suffices to show that

$$
\gamma-1: t^{k} \mathrm{D}_{\text {dif }}^{+, n}\left(D_{S}\right) / t^{k+1} \mathrm{D}_{\text {dif }}^{+, n}\left(D_{S}\right) \rightarrow t^{k} \mathrm{D}_{\text {dif }}^{+, n}\left(D_{S}\right) / t^{k+1} \mathrm{D}_{\text {dif }}^{+, n}\left(D_{S}\right)
$$

is bijective for any $k \geq b-a+1$. Note that $t^{k} \mathrm{D}_{\text {dif }}^{+, n}\left(D_{S}\right) / t^{k+1} \mathrm{D}_{\text {dif }}^{+, n}\left(D_{S}\right)$ is isomorphic to $\mathrm{D}_{\text {Sen }}^{n}\left(D_{S}(k)\right)$ as a $\Gamma$-module. Furthermore, note that $\mathrm{D}_{\text {Sen }}^{n}\left(D_{S}(k)\right)=$ $\bigoplus_{a \leq j \leq b}\left(\mathrm{D}_{\text {Sen }}^{n}\left(D_{S}\right)\right)^{\Gamma_{n}=\chi^{j+k}}$ by Lemma 3.8. Since $j+k \geq b+1$ for all $j \in[a, b]$, we deduce that $\gamma-\chi^{i}(\gamma)$ is bijective on $\mathrm{D}_{\text {Sen }}^{n}\left(D_{S}(k)\right)$.

Lemma 4.9. Let $D_{S}$ be a Hodge-Tate $(\varphi, \Gamma)$-module over $\boldsymbol{B}_{\mathrm{rig}, K}^{\dagger} \hat{\otimes}_{\mathbb{Q}_{p}} S$ with weights in $[a, b]$. Then $D_{S}$ is de Rham if and only if there exists a positive integer $n \geq h_{\mathrm{HT}}\left(D_{S}\right)$ such that $\prod_{i=a}^{2 b-a}\left(\gamma-\chi(\gamma)^{i}\right) \mathrm{D}_{\mathrm{dif}}^{+, n}\left(D_{S}\right) \subset t^{b-a+1} \mathrm{D}_{\mathrm{dif}}^{+, n}\left(D_{S}\right)$. Furthermore, if this is the case, then (4.1.1) holds for $n$.

Proof. Suppose that $D_{S}$ is de Rham. Let $n \geq h_{\mathrm{dR}}\left(D_{S}\right)$, and put

$$
N=D_{\mathrm{dR}}\left(D_{S}\right) \otimes_{K \otimes_{\mathbb{Q}_{p}} S}\left(K_{n} \otimes_{\mathbb{Q}_{p}} S\right) \llbracket t \rrbracket .
$$

Since $D$ has weights in $[a, b]$, by Lemma 4.7 we have $t^{-a} \mathrm{D}_{\text {dif }}^{+, n}\left(D_{S}\right) \subset N \subset$ $t^{-b} \mathrm{D}_{\mathrm{dif}}^{+, n}\left(D_{S}\right)$. On the other hand, by the construction of $N$, it is clear that $(\gamma-1) N \subset t N$. It therefore follows that

$$
\begin{aligned}
\prod_{i=a}^{2 b-a}\left(\gamma-\chi(\gamma)^{i}\right) \mathrm{D}_{\mathrm{dif}}^{+, n}\left(D_{S}\right) \subset \prod_{i=a}^{2 b-a}\left(\gamma-\chi(\gamma)^{i}\right)\left(t^{a} N\right) \\
\subset t^{2 b-a+1} N \subset t^{b-a+1} \mathrm{D}_{\mathrm{dif}}^{+, n}\left(D_{S}\right) .
\end{aligned}
$$

Now suppose $\prod_{i=a}^{2 b-a}\left(\gamma-\chi(\gamma)^{i}\right) \mathrm{D}_{\text {dif }}^{+, n}\left(D_{S}\right) \subset t^{b-a+1} \mathrm{D}_{\text {dif }}^{+, n}\left(D_{S}\right)$ for some $n \geq$ $h_{\mathrm{HT}}\left(D_{S}\right)$. We claim that for any $j \in[a, b]$ and $a \in\left(\mathrm{D}_{\mathrm{Sen}}^{n}\left(D_{S}\right)\right)^{\Gamma_{n}=\chi^{j}}$, we can lift $a$ to an element in $\left(\mathrm{D}_{\mathrm{dif}}^{+, n}\left(D_{S}\right)\right)^{\Gamma_{n}=\chi^{j}}$. In fact, let $\tilde{a}$ be any lift of $a$ in $\mathrm{D}_{\mathrm{dif}}^{+, n}\left(D_{S}\right)$, and let

$$
\tilde{b}=\prod_{\substack{a \leq i \leq 2 b-a \\ i \neq j}} \frac{\gamma-\chi^{i}(\gamma)}{\chi^{j}(\gamma)-\chi^{i}(\gamma)} \tilde{a},
$$

where $\gamma$ is a topological generator of $\Gamma_{n}$; it is clear that $\tilde{b}$ is also a lift of $a$. Furthermore, by assumption, we have $\left(\gamma-\chi^{j}(\gamma)\right)(\tilde{b}) \in \prod_{i=a}^{2 b-a}\left(\gamma-\chi(\gamma)^{i}\right) \mathrm{D}_{\mathrm{dif}}^{+, n}\left(D_{S}\right) \subset$ $t^{b-a+1} \mathrm{D}_{\mathrm{dif}}^{+, n}\left(D_{S}\right)$. By the previous lemma, we choose some $\tilde{c} \in t^{b-a+1} \mathrm{D}_{\text {dif }}^{+, n}\left(D_{S}\right)$ satisfying $\left(\gamma-\chi^{j}(\gamma)\right)(\tilde{b})=\left(\gamma-\chi^{j}(\gamma)\right)(\tilde{c})$. It is then clear that $\tilde{b}-\tilde{c}$ is a desired lift of $a$. Since $\mathrm{D}_{\text {Sen }}^{n}\left(D_{S}\right)=\bigoplus_{a \leq i \leq b}\left(\mathrm{D}_{\text {Sen }}^{n}\left(D_{S}\right)\right)^{\Gamma_{n}=\chi^{i}}$, we have that $\left(\mathrm{D}_{\text {Sen }}^{n}\left(D_{S}\right)\right)^{\Gamma_{n}=\chi^{i}}$ is locally free for each $i \in[a, b]$. By shrinking $M(S)$, we may further suppose that each $\left(\mathrm{D}_{\mathrm{Sen}}^{n}\left(D_{S}\right)\right)^{\Gamma_{n}=\chi^{i}}$ is free. We then deduce from the claim that there exists a free $K_{n} \otimes_{\mathbb{Q}_{p}} S$-module $M \subseteq\left(\mathrm{D}_{\text {dif }}^{n}\left(D_{S}\right)\right)^{\Gamma_{n}}$ such that the natural map

$$
M \otimes_{K_{n} \otimes_{\mathbb{Q}_{p}} S}\left(K_{n} \otimes_{\mathbb{Q}_{p}} S\right) \llbracket t \rrbracket[1 / t] \longrightarrow \mathrm{D}_{\mathrm{dif}}^{n}\left(D_{S}\right)
$$


is an isomorphism. It follows that the natural map

$$
M^{\Gamma} \otimes_{K \otimes_{\mathbb{Q}_{p}} S}\left(K_{n} \otimes_{\mathbb{Q}_{p}} S\right) \llbracket t \rrbracket[1 / t] \longrightarrow \mathrm{D}_{\mathrm{dif}}^{n}\left(D_{S}\right)
$$

is an isomorphism because $M=M^{\Gamma} \otimes_{K \otimes_{\mathbb{Q}_{p}} S}\left(K_{n} \otimes_{\mathbb{Q}_{p}} S\right)$ by [Berger and Colmez 2008, Proposition 2.2.1]. Taking $\Gamma$-invariants on both sides, we get $M^{\Gamma}=\left(\mathrm{D}_{\text {dif }}^{n}\left(D_{S}\right)\right)^{\Gamma}$. This implies that $D_{S}$ is de Rham.

Proposition 4.10. Let $S$ be reduced, and let $D_{S}$ be a $(\varphi, \Gamma)$-module over $\boldsymbol{B}_{\mathrm{rig}, K}^{\dagger} \hat{\otimes} S$. Suppose that there exists a Zariski-dense subset $Z \subset M(S)$ such that $D_{z}$ is de Rham with weights in $[a, b]$ for any $z \in Z$ and $\sup _{z \in Z}\left\{h_{\mathrm{dR}}\left(D_{z}\right)\right\}<\infty$. Then $D_{S}$ is de Rham with weights in $[a, b]$.

Proof. By Proposition 3.9, we first have that $D_{S}$ is Hodge-Tate with weights in $[a, b]$. Let $n \geq \max \left\{h_{\mathrm{HT}}\left(D_{S}\right), \sup _{z \in Z}\left\{h_{\mathrm{dR}}\left(D_{z}\right)\right\}\right\}$. By Lemma 4.9, we have

$$
\prod_{i=a}^{2 b-a}\left(\gamma-\chi(\gamma)^{i}\right) \mathrm{D}_{\mathrm{dif}}^{+, n}\left(D_{z}\right) \subset t^{b-a+1} \mathrm{D}_{\mathrm{dif}}^{+, n}\left(D_{z}\right)
$$

for any $z \in Z$. This implies $\prod_{i=a}^{2 b-a}\left(\gamma-\chi(\gamma)^{i}\right) \mathrm{D}_{\mathrm{dif}}^{+, n}\left(D_{S}\right) \subset t^{b-a+1} \mathrm{D}_{\mathrm{dif}}^{+, n}\left(D_{S}\right)$ because $S$ is reduced and $Z$ is Zariski-dense. Hence $D_{S}$ is de Rham by Lemma 4.9 again.

Remark 4.11. The work presented in this paper was finished in the summer of 2012 and made public at the beginning of 2013. Later that year came the preprint of [Bellovin 2015], in which the author built up a more robust theory of families of Hodge-Tate and de Rham representations over rigid analytic spaces. First of all, she generalized Berger's dictionary, which relates Fontaine's functors to $(\varphi, \Gamma)$ modules, to families of $p$-adic representations [Bellovin 2015, Theorem 1.1.1]. This result implies that our theory of families of Hodge-Tate and de Rham $(\varphi, \Gamma)$ modules with bounded Hodge-Tate weights developed in $\S 3$ and $\S 4$ can be viewed as a generalization of Berger and Colmez's theory of families of Hodge-Tate and de Rham representations with bounded Hodge-Tate weights. Moreover, she developed a theory of families of "partial" Hodge-Tate and de Rham representations with bounded Hodge-Tate weights. That is, the periods of the fibers are assumed to be of some constant rank which is not necessarily equal to the rank of the family. In addition, she removes the "reduced" assumption on the base by considering all artinian points. We refer the reader to [Bellovin 2015] for more results and details.

\section{5. $p$-adic local monodromy for families of de $\operatorname{Rham}(\varphi, \Gamma)$-modules}

The main goal of this section is to prove the $p$-adic local monodromy for the restrictions of families of de Rham $(\varphi, \Gamma)$-modules with bounded Hodge-Tate weights on their Shilov boundary. The proof is modeled on Berger and Colmez's 
proof of the $p$-adic local monodromy for families of de Rham representations with bounded Hodge-Tate weights [Berger and Colmez 2008, §6]. Recall that $\nabla=\log (\gamma) / \log (\chi(\gamma))$, which is independent of the choice of $\gamma \in \Gamma$. This gives rise to an action of the Lie algebra of $\Gamma$ on $(\varphi, \Gamma)$-modules and their localizations. In the following, we fix $E$ to be a finite extension of the products of the complete residue fields of the Shilov boundary of $M(S)$.

Proposition 5.1. Let $D_{S}$ be a de Rham $(\varphi, \Gamma)$-module of rank $d$ over $\boldsymbol{B}_{\mathrm{rig}, K}^{\dagger} \hat{\otimes}_{\mathbb{Q}_{p}} S$ with weights in $[a, b]$. For any $s>0$ such that $n(s) \geq h_{\mathrm{dR}}\left(D_{S}\right)$, let $N_{S}\left(D_{E}\right)=\left\{y \in t^{-b} D_{E}^{S}\right.$ such that $\iota_{n}(y) \in D_{\mathrm{dR}}\left(D_{S}\right) \otimes_{K \otimes_{\mathbb{Q}_{p}} S}\left(K_{n} \otimes_{\mathbb{Q}_{p}} E\right) \llbracket t \rrbracket$ for each $n \geq n(s)\}$.

Then the following are true:

(1) The $\boldsymbol{B}_{\mathrm{rig}, K}^{\dagger, s} \hat{\otimes}_{\mathbb{Q}_{p}} E$-module $N_{s}\left(D_{E}\right)$ is free of rank $d$ and stable under $\Gamma$.

(2) For each $n \geq n(s)$, we have

$N_{S}\left(D_{E}\right) \otimes_{\boldsymbol{B}_{\mathrm{rig}, K}^{\dagger, s}} \hat{\otimes}_{\mathbb{Q}_{p} E, l_{n}}\left(K_{n} \otimes_{\mathbb{Q}_{p}} E\right) \llbracket t \rrbracket=D_{\mathrm{dR}}\left(D_{S}\right) \otimes_{K \otimes_{\mathbb{Q}_{p}} S}\left(K_{n} \otimes_{\mathbb{Q}_{p}} E\right) \llbracket t \rrbracket$.

Furthermore, if we put $N_{\mathrm{dR}}\left(D_{E}\right)=N_{S}\left(D_{E}\right) \otimes_{\boldsymbol{B}_{\mathrm{rig}, K}^{\dagger, s} \hat{\otimes}_{\mathbb{Q}_{p}} E} \boldsymbol{B}_{\mathrm{rig}, K}^{\dagger} \widehat{\otimes}_{\mathbb{Q}_{p}} E$, then the following are true:

(3) The $\boldsymbol{B}_{\mathrm{rig}, K}^{\dagger} \hat{\otimes}_{\mathbb{Q}_{p}}$ E-module $N_{\mathrm{dR}}\left(D_{E}\right)$ is free of rank $d$, stable under $\Gamma$, and independent of the choice of $s$.

(4) We have $\varphi^{*}\left(N_{\mathrm{dR}}\left(D_{E}\right)\right)=N_{\mathrm{dR}}\left(D_{E}\right)$ and $\nabla\left(N_{\mathrm{dR}}\left(D_{E}\right)\right) \subset t \cdot N_{\mathrm{dR}}\left(D_{E}\right)$.

Proof. First, note that the sequence of $K_{n} \otimes_{\mathbb{Q}_{p}} E \llbracket t \rrbracket$-modules

$$
\left\{D_{\mathrm{dR}}\left(D_{S}\right) \otimes_{K \otimes_{\mathbb{Q} p} S}\left(K_{n} \otimes_{\mathbb{Q}_{p}} E\right) \llbracket t \rrbracket\right\}_{n \geq n(s)}
$$

is $\varphi$-compatible in the sense of [Berger 2008b, Définition II.1.1]. Then by the proof of [Berger 2008b, Théorème II.1.2] (using the fact that $E$ is a finite product of $p$ adic local fields which are endowed with discrete valuations extending the standard one on $\left.\mathbb{Q}_{p}\right)$, we see that $N_{\mathrm{dR}}\left(D_{E}\right)$ is the unique $(\varphi, \Gamma)$-module $M_{E}$ contained in $D_{E}[1 / t]$ such that

$$
M_{E}^{S} \otimes_{\boldsymbol{B}_{\mathrm{rig}, K}^{\dagger, s} \hat{\otimes}_{\mathbb{Q}_{p}} E, \iota_{n}}\left(K_{n} \otimes_{\mathbb{Q}_{p}} E\right) \llbracket t \rrbracket=D_{\mathrm{dR}}\left(D_{S}\right) \otimes_{K \otimes_{\mathbb{Q}_{p}} S}\left(K_{n} \otimes_{\mathbb{Q}_{p}} E\right) \llbracket t \rrbracket
$$

for any $n \geq n(s)$. Furthermore, the proof of [Berger 2008b, Théorème II.1.2] implies all of the proposition except the second half of (4). To see that part, note that

$$
\iota_{n}\left(\nabla\left(N_{S}\left(D_{E}\right)\right)\right)=\nabla\left(\iota_{n}\left(N_{S}\left(D_{E}\right)\right)\right) \subset t D_{\mathrm{dR}}\left(D_{S}\right) \otimes_{K \otimes_{\mathbb{Q}_{p}} S}\left(K_{n} \otimes_{\mathbb{Q}_{p}} E\right) \llbracket t \rrbracket .
$$

This yields that $\nabla\left(N_{S}\left(D_{E}\right)\right) \subset t N_{S}\left(D_{E}\right)$ for all $s$. Thus $\nabla\left(N_{\mathrm{dR}}\left(D_{E}\right)\right) \subset t N_{\mathrm{dR}}\left(D_{E}\right)$, as $N_{\mathrm{dR}}\left(D_{E}\right)$ is equal to the union of all $N_{S}\left(D_{E}\right)$. 
Proposition 5.2. Keep the notation of Proposition 5.1. Then there exists a finite extension $L$ over $K$ such that

$$
M=\left(N_{\mathrm{dR}}\left(D_{E}\right) \otimes_{\boldsymbol{B}_{\mathrm{rig}, K}^{\dagger}} \hat{\otimes}_{\mathbb{Q}_{p}} E \boldsymbol{B}_{\log , L}^{\dagger} \hat{\otimes}_{\mathbb{Q}_{p}} E\right)^{I_{L}}
$$

is a free $L_{0}^{\prime} \otimes_{\mathbb{Q}_{p}} E$-module of rank $d$ and the natural map

$$
M \otimes_{L_{0}^{\prime} \otimes_{\mathbb{Q}_{p}} E} \boldsymbol{B}_{\log , L}^{\dagger} \widehat{\otimes}_{\mathbb{Q}_{p}} E \longrightarrow N_{\mathrm{dR}}\left(D_{E}\right) \otimes_{\boldsymbol{B}_{\mathrm{rg}, K}^{\dagger} \widehat{\otimes}_{\mathbb{Q}_{p}} E} \boldsymbol{B}_{\log , L}^{\dagger} \widehat{\otimes}_{\mathbb{Q}_{p}} E
$$

is an isomorphism.

Proof. Let $f^{\prime}=\left[K_{0}^{\prime}: \mathbb{Q}_{p}\right]$. Note that there is a canonical decomposition

$$
\boldsymbol{B}_{\mathrm{rig}, K}^{\dagger} \hat{\otimes}_{\mathbb{Q}_{p}} E \cong \prod_{i=0}^{f^{\prime}-1} \mathscr{R}_{E}^{(i)},
$$

where each $\mathscr{R}_{E}^{(i)}$ is isomorphic to $\mathscr{R}_{E}$ and stable under $\Gamma_{K}$, and satisfies $\varphi\left(\mathscr{R}_{E}^{(i)}\right) \subset$ $\mathscr{R}_{E}^{(i+1)}$ (define $\left.\mathscr{R}_{E}^{\left(f^{\prime}\right)}=\mathscr{R}_{E}^{(0)}\right)$. Let

$$
N_{\mathrm{dR}}^{(i)}\left(D_{E}\right)=N_{\mathrm{dR}}\left(D_{E}\right) \otimes_{\boldsymbol{B}_{\mathrm{rig}, K}^{\dagger}} \widehat{\otimes}_{\mathbb{Q}_{p}} E \mathscr{R}_{E}^{(i)} .
$$

It follows that each $N_{\mathrm{dR}}^{(i)}\left(D_{E}\right)$ is stable under $\partial=\nabla / t$ and $\varphi^{f^{\prime}}$; hence it is a $p$-adic differential equation with a Frobenius structure. By the versions of the $p$-adic local monodromy theorem proved by André [2002] or Mebkhout [2002], we conclude that each $N_{\mathrm{dR}}^{(i)}\left(D_{E}\right)$ is potentially unipotent. This yields the proposition using the argument of Proposition 6.2.2 and Corollaire 6.2.3 of [Berger and Colmez 2008].

Lemma 5.3. Keep notation as in Proposition 5.2, and let

$$
M=\left(N_{s}\left(D_{E}\right) \otimes_{\boldsymbol{B}_{\mathrm{rig}, K}^{\dagger, s} \hat{\otimes}_{\mathbb{Q}_{p}} E} \boldsymbol{B}_{\log , K}^{\dagger, s} \widehat{\otimes}_{\mathbb{Q}_{p}} E\right)^{I_{L}}
$$

for sufficiently large $s$. Then, for any $n \geq n(s)$, we have

$$
L \otimes_{L_{0}} \iota_{n}(M)=\left(\mathrm{D}_{\mathrm{dif}}\left(D_{E} \otimes_{\boldsymbol{B}_{\mathrm{rig}, K}^{\dagger} \hat{\otimes}_{\mathbb{Q}_{p}} E} \boldsymbol{B}_{\mathrm{rig}, L}^{\dagger} \hat{\otimes}_{\mathbb{Q}_{p}} E\right)\right)^{I_{L}} .
$$

Proof. By the previous proposition, the left side of (5.3.1) is a free $L \otimes_{L_{0}} L_{0}^{\prime} \otimes_{\mathbb{Q}_{p}} E$ module of rank $d$. On the other hand, since $\left(\left(L_{n} \otimes_{\mathbb{Q}_{p}} E\right) \llbracket t \rrbracket[1 / t]\right)^{I_{L}}=L \otimes_{L_{0}}$ $L_{0}^{\prime} \otimes_{\mathbb{Q}_{p}} E$, we deduce that the right side of (5.3.1), which obviously contains the left side, is an $L \otimes_{L_{0}} L_{0}^{\prime} \otimes_{\mathbb{Q}_{p}} E$-module generated by at most $d$ elements. Using the fact that $L \otimes_{L_{0}} L_{0}^{\prime} \otimes_{\mathbb{Q}_{p}} E$ is a product of fields, we deduce the desired identity. 


\section{Proof of the main theorem}

Now let $V_{X}$ be a finite slope family of dimension $d$ over a reduced rigid analytic space $X$ over $F$. We start by making some preliminary reductions. After a finite surjective base change of $X$, we may assume that $Q(T)$ factors as $\prod_{i=1}^{m}\left(T-F_{i}\right)$. By reordering the $F_{i}$ and throwing away some points of $Z$, we may further assume that, for all $z \in Z, v_{p}\left(F_{i}(z)\right) \geq v_{p}\left(F_{j}(z)\right)$ if $i>j$ and $F_{i}(z) \neq F_{j}(z)$ if $F_{i} \neq F_{j}$. We then set $\mathscr{F}_{i, z}=D_{\text {st }}^{+}\left(V_{z}\right)^{\left(\varphi^{f}-F_{1}(z)\right) \cdots\left(\varphi^{f}-F_{i}(z)\right)=0}$ for all $z \in Z$ and $1 \leq i \leq m$. Using Definition 0.1(3), we may suppose that $\mathscr{F}_{i, z} \subseteq \mathscr{F}_{z}$ for all $z \in Z$ and $1 \leq i \leq m$ by shrinking $Z$. Furthermore, by the fact that $N \varphi=p \varphi N$ and the condition that $v_{p}\left(F_{i}(z)\right) \geq v_{p}\left(F_{j}(z)\right)$ if $i>j$, we see that $N=0$ on each graded piece $\mathscr{F}_{i, z} / \mathscr{F}_{i-1, z}$. Let $c_{i, z}$ be the rank of $\mathscr{F}_{i, z} / \mathscr{F}_{i-1, z}$ over $K_{0} \otimes k(z)$, and partition $Z$ into finitely many subsets according to the sequence $c_{i, z}$. Note that at least one of these subsets of $Z$ has to be Zariski-dense. Replace $Z$ by this subset, and set $c_{i}=c_{i, z}$ for $z \in Z$.

For $z \in Z$, we will inductively define $(\varphi, \Gamma)$-submodules Fil $_{i, z} \subset \mathrm{D}_{\text {rig }}^{\dagger}\left(V_{z}\right)$ for $1 \leq i \leq m$ such that $D_{\text {st }}^{+}\left(\operatorname{Fil}_{i, z}\right)=\mathscr{F}_{i, z}$. For $i=1$, since $V_{z}$ has nonpositive Hodge-Tate weights and $N\left(\mathscr{F}_{1, z}\right)=0$, we have

$$
\mathscr{F}_{1, z}=D_{\mathrm{st}}^{+}\left(V_{z}\right)^{\varphi^{f}=F_{1}(z), N=0}=D_{\text {crys }}^{+}\left(V_{z}\right)^{\varphi^{f}=F_{1}(z)}=\mathrm{D}_{\mathrm{rig}}^{\dagger}\left(V_{z}\right)^{\Gamma=1, \varphi^{f}=F_{z}(z)},
$$

using Berger's dictionary [2002, Théorème 3.6]. Let $\mathrm{Fil}_{1, z}$ be the saturation of the $(\varphi, \Gamma)$-submodule of $\mathrm{D}_{\text {rig }}^{\dagger}\left(V_{z}\right)$ generated by $\mathscr{F}_{1, z}$. It is then clear that $D_{\text {st }}^{+}\left(F_{1} i_{1, z}\right)=$ $D_{\text {crys }}^{+}\left(\right.$Fil $\left._{1, z}\right)=\mathscr{F}_{1, z}$. Now suppose we have defined Fil ${ }_{i-1, z}$ for some $i \geq 2$ such that $D_{\text {st }}^{+}\left(\operatorname{Fil}_{i-1, z}\right)=\mathscr{F}_{i-1, z}$. It follows that

$$
D_{\text {st }}^{+}\left(\mathrm{D}_{\text {rig }}^{\dagger}\left(V_{z}\right) / \operatorname{Fil}_{i-1, z}\right)=D_{\text {st }}^{+}\left(V_{z}\right) / \mathscr{F}_{i-1, z} .
$$

Note that

$$
\mathscr{F}_{i, z} / \mathscr{F}_{i-1, z}=\left(D_{\mathrm{st}}^{+}\left(V_{z}\right) / \mathscr{F}_{i-1, z}\right)^{\varphi^{f}=F_{i}(z), N=0} .
$$

Hence

$$
\mathscr{F}_{i, z} / \mathscr{F}_{i-1, z}=D_{\text {crys }}^{+}\left(\mathrm{D}_{\text {rig }}^{\dagger}\left(V_{z}\right) / \operatorname{Fil}_{i, z}\right)^{\varphi^{f}=F_{i}(z)} \subset\left(\mathrm{D}_{\text {rig }}^{\dagger}\left(V_{z}\right) / \operatorname{Fil}_{i-1, z}\right)^{\Gamma} .
$$

We set $\mathrm{Fil}_{i, z}$ to be the preimage of the saturation of the $(\varphi, \Gamma)$-submodule of $\mathrm{D}_{\text {rig }}^{\dagger}\left(V_{z}\right) /$ Fil $_{i-1, z}$ generated by $\mathscr{F}_{i, z} / \mathscr{F}_{i-1, z}$. Now, for each $1 \leq i \leq m$, we define the character $\delta_{i}: K^{\times} \rightarrow \mathcal{O}(X)^{\times}$by setting $\delta_{i}(p)=F_{i}^{-1}$ and $\delta_{i}\left(\mathcal{O}_{K}^{\times}\right)=1$. Let $D_{X}=\mathrm{D}_{\text {rig }}^{\dagger}\left(V_{X}\right)^{\vee}$.

Lemma 6.1. Suppose that $X$ is irreducible. Then, for each $0 \leq i \leq m$, there exists a proper birational morphism $\pi: X^{\prime} \rightarrow X$ and a subfamily of $(\varphi, \Gamma)$-modules $D_{X^{\prime}}^{(i)} \subset D_{X^{\prime}}$ over $X^{\prime}$ of rank $d-c_{1}-\cdots-c_{i}$ such that:

(1) For any $x \in X^{\prime}$, the natural map $D_{x}^{(i)} \rightarrow D_{x}$ is injective. 
(2) There exists a Zariski-open dense subset $U$ of $X^{\prime}$ such that for any $z \in Z^{\prime}=$ $\pi^{-1}(Z) \cap U$, the natural map $D_{z}^{(i)} \rightarrow D_{z}$ is the dual of the projection $\mathrm{D}_{\text {rig }}^{\dagger}\left(V_{\pi(z)}\right) \rightarrow \mathrm{D}_{\text {rig }}^{\dagger}\left(V_{\pi(z)}\right) / \operatorname{Fil}_{i, \pi(z)}$.

Proof. We proceed by induction on $i$. The initial case is trivial. Suppose that, for some $1 \leq i \leq m$, the lemma is true for $i-1$. Note that $\mathscr{F}_{i, z} / \mathscr{F}_{i-1, z}$ maps into $\mathrm{D}_{\text {rig }}^{\dagger}\left(V_{z}\right) / \mathrm{Fil}_{i, z}$ for any $z \in Z$. Since $\mathscr{F}_{i, z} / \mathscr{F}_{i-1, z}=\left(D_{\text {crys }}^{+}\left(V_{z}\right) / \mathscr{F}_{i-1, z}\right)^{\varphi^{f}=F_{i}(z)}$, we get that $\left(D_{z}^{(i)}\right)^{\vee}\left(\pi^{*}\left(\delta_{i}\right)(z)\right)$ has $k(z)$-dimension $c_{i}$ for any $z \in Z^{\prime}$. Since $Z^{\prime}$ is Zariski-dense in $X^{\prime}$, by Proposition 2.5 after adapting $X^{\prime}$ and $U$, we may find a subfamily of $(\varphi, \Gamma)$-modules $D_{X^{\prime}}^{(i)}$ of $D_{X^{\prime}}^{(i-1)}$ with rank $d-c_{1}-\cdots-c_{i}$ such that:

(1') $D_{x}^{(i)} \rightarrow D_{x}^{(i-1)}$ is injective for any $x \in X^{\prime}$.

(2') For any $z \in \pi^{-1}(Z) \cap U, D_{z}^{(i)}$ is the kernel of the dual of the map

$$
\left(\boldsymbol{B}_{\mathrm{rig}, K}^{\dagger} \otimes_{\mathbb{Q}_{p}} k(z)\right) \cdot\left(\mathscr{F}_{i, \pi(z)} / \mathscr{F}_{i-1, \pi(z)}\right) \rightarrow \mathrm{D}_{\mathrm{rig}}^{\dagger}\left(V_{\pi(z)}\right) / \mathrm{Fil}_{i, \pi(z)} .
$$

It is clear that (1') and (2') imply (1) and (2), respectively; this finishes the inductive step.

To prove Theorem 0.3, we also need the following lemma.

Lemma 6.2. Let $V_{S}$ be a free $S$-linear representation of $G_{K}$ of rank $d$. Then there exists a positive integer $m\left(V_{S}\right)$ such that for any $x \in M(S)$ and $a \in \mathrm{D}_{\mathrm{dif}}^{+}\left(V_{x}\right)$, if $a$ is $\Gamma$-invariant, then $a \in \mathrm{D}_{\mathrm{dif}}^{+, m\left(V_{S}\right)}\left(V_{x}\right)$.

Proof. This is a consequence of the Tate-Sen method. Using [Berger and Colmez 2008, Théorème 4.2.9], we first choose a finite extension $L$ over $K$ and some positive integer $m$ so that $\mathrm{D}_{\mathrm{rig}, L}^{\dagger, r_{m}}\left(V_{S}\right)$ is a free $\boldsymbol{B}_{\mathrm{rig}, L}^{\dagger, r_{m}} \hat{\otimes}_{\mathbb{Q}_{p}} S$-module with a basis $\boldsymbol{e}=\left(e_{1}, \ldots, e_{d}\right)$. Let $\gamma$ be a topological generator of $\Gamma_{L_{m}}$ and write $\gamma(e)=e G$ for some $G \in \mathrm{GL}_{d}\left(\boldsymbol{B}_{\mathrm{rig}, L}^{\dagger, r_{m}} \hat{\otimes}_{\mathbb{Q}_{p}} S\right)$. Recall that by the classical work [Tate 1967] we know that there exists a constant $c>0$ such that $v_{p}((\gamma-1) x) \leq v_{p}(x)+c$ for any nonzero $x \in\left(1-R_{L, m}\right) \hat{L}_{\infty}$, where $R_{L, m}: \hat{L}_{\infty} \rightarrow L_{m}$ is Tate's normalized trace map. Since the localization map $\iota_{m}: \boldsymbol{B}_{\text {rig, }, L}^{\dagger, r_{m}} \rightarrow L_{m} \llbracket t \rrbracket$ is continuous, by enlarging $m$ we may suppose that the constant term of $\iota_{m}(G)-1$ has norm less than $p^{-c}$. We fix some $m_{0} \in \mathbb{N}$ such that $K_{\infty} \cap L_{m}=K_{m_{0}} \cap L_{m}$.

Now let $a \in \mathrm{D}_{\text {dif }}^{+, K_{n}}\left(V_{x}\right)^{\Gamma}$ for some $x \in X$ and $n \geq m$. We will show that $a \in \mathrm{D}_{\mathrm{dif}}^{+, K_{m_{0}}}\left(V_{x}\right)^{\Gamma}$. Since $\iota_{m}(\boldsymbol{e})$ forms a basis of $\mathrm{D}_{\mathrm{dif}}^{+, L_{n}}\left(V_{S}\right)$, we may write $a=$ $\iota_{m}(e)(x) A$ for some

$$
A \in \mathrm{M}_{d \times 1}\left(\left(L_{n} \otimes_{\mathbb{Q}_{p}} k(x)\right) \llbracket t \rrbracket\right) .
$$

The $\Gamma$-invariance of $a$ implies $\iota_{m}(G(x)) \gamma(A)=A$; thus

$$
\left(1-R_{L, m}\right) \iota_{m}(G(x)) \gamma(A)=\left(1-R_{L, m}\right) A
$$


Note that $\iota_{m}(G(x))$ has entries in $\left(L_{m} \otimes_{\mathbb{Q}_{p}} k(x)\right) \llbracket t \rrbracket$. It follows that $(G(x)-1) B=$ $\left(1-\gamma^{-1}\right) B$, where $B=\left(1-R_{L, m}\right) A$. Let $B_{0}$ be the constant term of $B$. If $B_{0} \neq 0$, then the constant term of $\left(\iota_{m}(G(x))-1\right) B$ has valuation

$$
\geq v\left(l_{m}(G(x))-1\right)+v\left(B_{0}\right)>v\left(B_{0}\right)+c,
$$

whereas the constant term $\left(1-\gamma^{-1}\right) B_{0}$ of $\left(1-\gamma^{-1}\right) B$ has valuation $\leq v\left(B_{0}\right)+c$; this yields a contradiction. Hence $B_{0}=0$. Iterating this argument, we get $B=0$. Hence $a \in \mathrm{D}_{\text {dif }}^{+, L_{m}}\left(V_{x}\right) \cap \mathrm{D}_{\text {dif }}^{+, K_{n}}\left(V_{x}\right) \subset \mathrm{D}_{\text {dif }}^{+, K_{m}}\left(V_{x}\right)$. Thus we may choose $m\left(V_{S}\right)=m_{0}$.

Remark 6.3. Although we do not need it in this paper, it is worthwhile to point out that the argument of Lemma 6.2 works equally well for families of $(\varphi, \Gamma)$ modules and even a sequence of $\varphi$-compatible $K_{n} \llbracket t \rrbracket \widehat{\otimes}_{\mathbb{Q}_{p}} S$-modules $\left\{M_{n}\right\}_{n}$ in the vein of [Berger 2008b, Définition II.1.1]. That is, each $M_{n}$ is a finite projective $K_{n} \llbracket t \rrbracket \hat{\otimes}_{\mathbb{Q}_{p}} S$-module equipped with a continuous $K_{n} \llbracket t \rrbracket$-semilinear and $S$-linear $\Gamma$-action, and satisfies $M_{n} \otimes_{K_{n} \llbracket t \rrbracket \hat{\otimes}_{\mathbb{Q}_{p}} S} K_{n+1} \llbracket t \rrbracket \widehat{\otimes}_{\mathbb{Q}_{p}} S \cong M_{n+1}$.

Proof of Theorem 0.3. We retain the notation above. By passing to irreducible components, we may suppose that $X$ is irreducible. We then apply Lemma 6.1 to $V_{X}$. Note that $V_{X^{\prime}}$ is again a finite slope family over $X^{\prime}$ with the Zariskidense set of semistable points $\pi^{-1}(Z)$. We may suppose that $X^{\prime}=X$. Let $\lambda: \mathrm{D}_{\text {rig }}^{\dagger}\left(V_{X}\right)=D_{X}^{\vee} \rightarrow\left(D_{X}^{(m)}\right)^{\vee}$ be the dual of $D_{X}^{(m)} \rightarrow D_{X}$, and let $P_{X}=\operatorname{ker}(\lambda)$. For any $x \in X$, since $D_{x}^{(m)} \rightarrow D_{x}$ is injective, we get that the image of $\lambda_{x}$ is a $(\varphi, \Gamma)$-submodule of rank $d-c_{1}-\cdots-c_{m}$. Thus, by Lemma 2.4, after adapting $X$ we may assume that $P_{X}$ is a family of $(\varphi, \Gamma)$-modules of rank $c_{1}+\cdots+c_{m}$, and there exists a Zariski-open dense subset $U \subset X$ such that $P_{x}=\operatorname{ker}\left(\lambda_{x}\right)$ for any $x \in U$. Note that $\operatorname{ker}\left(\lambda_{z}\right)=\operatorname{Fil}_{i, z}$ for any $z \in Z$. Thus, by replacing $Z$ with $Z \cap U$, we may assume that $P_{z}=$ Fil $_{i, z}$ for any $z \in Z$. We claim that $P_{X}$ is de Rham with weights in $[-b, 0]$. To do so, we set $Y$ to be the set of $x \in X$ for which $P_{x}$ is de Rham with weights in $[a, b]$. By the previous lemma, we see that for any affinoid subdomain $M(S) \subset X$, there exists an integer $m\left(V_{S}\right)$ such that if $P_{x}$ is de Rham for some $x \in M(S)$, then $h_{\mathrm{dR}}\left(P_{x}\right) \leq m\left(V_{S}\right)$. We then deduce from Proposition 4.10 that $Y \cap M(S)$ is a Zariski-closed subset of $M(S)$. Hence $Y$ is a Zariski-closed subset of $X$. On the other hand, since $P_{z}$ is de Rham with weights in $[-b, 0]$, we get $Z \subset Y$; thus $Y=X$ by the Zariski density of $Z$. Furthermore, using Proposition 4.10 and the previous lemma again, we deduce that $P_{X}$ is de Rham with weights in $[-b, 0]$. As a consequence, we obtain a locally free coherent $\mathcal{O}_{X} \otimes_{\mathbb{Q}_{p}} K$-module $D_{\mathrm{dR}}\left(P_{X}\right)$ of rank $c_{1}+\cdots+c_{m}$.

The next step is to show that $D_{\mathrm{dR}}\left(P_{x}\right)$ is contained in $D_{\mathrm{st}}^{+}\left(V_{x}\right) \otimes_{K_{0}} K$ for any $x \in X$. Let $Y$ be the set of $x \in X$ satisfying this condition. We first show that $Y$ is a 
Zariski-closed subset of $X$. For this, it suffices to show that $Y \cap M(S)$ is a Zariskiclosed subset of $M(S)$ for any affinoid subdomain $M(S)$ of $X$. To show this, we employ the $p$-adic local monodromy for families of de Rham $(\varphi, \Gamma)$-modules. As in $\S 5$, let $E$ be the product of the complete residue fields of the Shilov boundary of $M(S)$. Since $P_{S}$ is a family of de $\operatorname{Rham}(\varphi, \Gamma)$-modules with weights in $[-b, 0]$, by Lemma 5.3 there exists a finite extension $L$ of $K$ such that for sufficiently large $s$ and $n \geq n(s)$, we have

$$
L \otimes_{L_{0}} \iota_{n}(M)=\left(\mathrm{D}_{\mathrm{dif}}\left(P_{E} \otimes_{\boldsymbol{B}_{\mathrm{rig}, K}^{\dagger} \hat{\otimes}_{\mathbb{Q}_{p}} E} \boldsymbol{B}_{\mathrm{rig}, L}^{\dagger} \hat{\otimes}_{\mathbb{Q}_{p}} E\right)\right)^{I_{L}}
$$

for $M=\left(N_{s}\left(P_{E}\right) \otimes_{\boldsymbol{B}_{\mathrm{rig}, K}^{\dagger, s} \hat{\otimes}_{\mathbb{Q}_{p}} E} \boldsymbol{B}_{\log , K}^{\dagger, s} \hat{\otimes}_{\mathbb{Q}_{p}} E\right)^{I_{L}}$; furthermore, $N_{s}\left(P_{E}\right) \subset P_{E}^{s}$. Thus

$$
\begin{aligned}
\iota_{n}(M) \subset \iota_{n}\left(P_{E} \otimes_{\boldsymbol{B}_{\mathrm{rig}, K}^{\dagger, s}} \hat{\otimes}_{\mathbb{Q}_{p} E} E\right. & \left.\boldsymbol{B}_{\log , K}^{\dagger, s} \hat{\otimes}_{\mathbb{Q}_{p}} E\right) \\
& \subset \iota_{n}\left(\mathrm{D}_{\mathrm{rig}}^{\dagger}\left(V_{E}\right) \otimes_{\boldsymbol{B}_{\mathrm{rig}, K}^{\dagger, s} \hat{\otimes}_{\mathbb{Q}_{p}} E} \boldsymbol{B}_{\log , K}^{\dagger, s} \hat{\otimes}_{\mathbb{Q}_{p}} E\right) \subset \boldsymbol{B}_{\mathrm{st}}^{+} \hat{\otimes}_{\mathbb{Q}_{p}} V_{E} .
\end{aligned}
$$

Note that $D_{\mathrm{dR}}\left(P_{E}\right) \subset \mathrm{D}_{\mathrm{dif}}^{+}\left(P_{E}\right) \subset \mathrm{D}_{\mathrm{dif}}^{+}\left(V_{E}\right) \subset \boldsymbol{B}_{\mathrm{dR}}^{+} \widehat{\otimes}_{\mathbb{Q}_{p}} V_{E}$. This yields

$$
D_{\mathrm{dR}}\left(P_{E}\right) \subset\left(\boldsymbol{B}_{\mathrm{st}}^{+} \hat{\otimes}_{\mathbb{Q}_{p}} V_{E}\right) \otimes_{L_{0}} L \cap \boldsymbol{B}_{\mathrm{dR}}^{+} \hat{\otimes}_{\mathbb{Q}_{p}} V_{E}=\left(\boldsymbol{B}_{\mathrm{st}}^{+} \hat{\otimes}_{\mathbb{Q}_{p}} V_{E}\right) \otimes_{L_{0}} L .
$$

We therefore deduce from [Berger and Colmez 2008, Lemme 6.3.1] that

$$
D_{\mathrm{dR}}\left(P_{S}\right) \subset\left(\boldsymbol{B}_{\mathrm{st}}^{+} \hat{\otimes}_{\mathbb{Q}_{p}} V_{E}\right) \otimes_{L_{0}} L \cap \boldsymbol{B}_{\mathrm{dR}}^{+} \hat{\otimes}_{\mathbb{Q}_{p}} V_{S}=\left(\boldsymbol{B}_{\mathrm{st}}^{+} \hat{\otimes}_{\mathbb{Q}_{p}} V_{S}\right) \otimes_{L_{0}} L .
$$

It follows that $Y \cap M(S)$, which is the set of $x \in M(S)$ such that $D_{\mathrm{dR}}\left(P_{x}\right) \subset$ $\left(\boldsymbol{B}_{\mathrm{st}}^{+} \otimes_{\mathbb{Q}_{p}} V_{x}\right) \otimes_{K_{0}} K$, is Zariski-closed in $M(S)$.

To conclude the proof of the theorem, it then suffices to show that, for any $x \in X$, $D_{\mathrm{dR}}\left(P_{x}\right) \subset\left(D_{\mathrm{st}}^{+}\left(V_{x}\right) \otimes_{K_{0}} K\right)^{Q\left(\varphi^{f}\right)(x)=0}$; here, we $K$-linearly extend the $\varphi^{f}$-action to $D_{\text {st }}^{+}\left(V_{x}\right) \otimes_{K_{0}} K$. Note that Fil ${ }_{m, z}$ is semistable with $D_{\text {st }}\left(\right.$ Fil $\left._{m, z}\right)=\mathscr{F}_{m, z}$. This implies that $Q\left(\varphi^{f}\right)\left(D_{\mathrm{dR}}\left(P_{X}\right)\right)$ vanishes at $z$, yielding that $Q\left(\varphi^{f}\right)\left(D_{\mathrm{dR}}\left(P_{X}\right)\right)=0$ by the Zariski density of $Z$.

\section{Acknowledgements}

Thanks to Christopher Skinner, Richard Taylor and Ila Varma for useful communications. We especially thank Richard Taylor for suggesting a more concise definition of finite slope families.

\section{References}

[André 2002] Y. André, "Filtrations de type Hasse-Arf et monodromie p-adique", Invent. Math. 148:2 (2002), 285-317. MR 2003k:12011 Zbl 1081.12003

[Bellaïche and Chenevier 2009] J. Bellaïche and G. Chenevier, Families of Galois representations and Selmer groups, Astérisque 324, 2009. MR $2011 \mathrm{~m}: 11105$ Zbl 1192.11035 
[Bellovin 2015] R. Bellovin, " $p$-adic Hodge theory in rigid analytic families", Algebra Number Theory 9:2 (2015), 371-433.

[Berger 2002] L. Berger, "Représentations p-adiques et équations différentielles", Invent. Math. 148:2 (2002), 219-284. MR 2004a:14022 Zbl 1113.14016

[Berger 2008a] L. Berger, "Construction de $(\phi, \Gamma)$-modules: représentations $p$-adiques et $B$-paires", Algebra Number Theory 2:1 (2008), 91-120. MR 2009j:14025 Zbl 1219.11078

[Berger 2008b] L. Berger, "Équations différentielles $p$-adiques et $(\phi, N)$-modules filtrés", pp. 13-38 in Représentations p-adiques de groupes p-adiques, I: Représentations galoisiennes et $(\phi, \Gamma)$ modules, edited by L. Berger et al., Astérisque 319, 2008. MR 2010d:11056 Zbl 1168.11019

[Berger and Colmez 2008] L. Berger and P. Colmez, "Familles de représentations de de Rham et monodromie $p$-adique", pp. 303-337 in Représentations p-adiques de groupes p-adiques, I: Représentations galoisiennes et $(\phi, \Gamma)$-modules, edited by L. Berger et al., Astérisque 319, 2008. MR 2010g:11091 Zbl 1168.11020

[Harris et al. 2014] M. Harris, K.-W. Lan, R. Taylor, and J. Thorne, "On the rigid cohomology of certain Shimura varieties”, preprint, 2014. arXiv 1411.6717

[Kedlaya and Liu 2010] K. Kedlaya and R. Liu, "On families of $(\phi, \Gamma)$-modules”, Algebra Number Theory 4:7 (2010), 943-967. MR 2012h:11081 Zbl 1278.11060

[Kedlaya et al. 2014] K. S. Kedlaya, J. Pottharst, and L. Xiao, "Cohomology of arithmetic families of $(\varphi, \Gamma)$-modules”, J. Amer. Math. Soc. 27:4 (2014), 1043-1115. MR 3230818 Zbl 06355531

[Kisin 2003] M. Kisin, "Overconvergent modular forms and the Fontaine-Mazur conjecture", Invent. Math. 153:2 (2003), 373-454. MR 2004f:11053 Zbl 1045.11029

[Liu 2014] R. Liu, “Triangulation of refined families", preprint, 2014. arXiv 1202.2188

[Mebkhout 2002] Z. Mebkhout, "Analogue $p$-adique du théorème de Turrittin et le théorème de la monodromie $p$-adique”, Invent. Math. 148:2 (2002), 319-351. MR 2003k:14018 Zbl 1071.12004

[Shah 2013] S. Shah, "Interpolating periods", preprint, 2013. arXiv 1305.2872

[Skinner and Urban 2006] C. Skinner and E. Urban, "Vanishing of $L$-functions and ranks of Selmer groups", pp. 473-500 in International Congress of Mathematicians, Volume II, edited by M. SanzSolé et al., Eur. Math. Soc., Zürich, 2006. MR 2008a:11063 Zbl 1157.11020

[Tate 1967] J. T. Tate, “p-divisible groups", pp. 158-183 in Proceedings of a Conference on Local Fields (Driebergen, 1966), edited by T. Springer, Springer, Berlin, 1967. MR 38 \#155 Zbl 0157.27601

Communicated by Brian Conrad

Received 2014-02-11 Revised 2014-12-09 Accepted 2015-01-14

liuruochuan@math.pku.edu.cn Beijing International Center for Mathematical Research, Peking University, Beijing, 100871, China 


\section{Algebra \& Number Theory}

msp.org/ant

\section{EDITORS}

MANAGING EDITOR

Bjorn Poonen

Massachusetts Institute of Technology

Cambridge, USA

\author{
EDITORIAL BOARD CHAIR \\ David Eisenbud \\ University of California \\ Berkeley, USA
}

BOARD OF EDITORS

Georgia Benkart

Dave Benson

Richard E. Borcherds

John H. Coates

J-L. Colliot-Thélène

Brian D. Conrad

Hélène Esnault

Hubert Flenner

Edward Frenkel

Andrew Granville

Joseph Gubeladze

Roger Heath-Brown

Craig Huneke

János Kollár

Yuri Manin

Barry Mazur

Philippe Michel

Susan Montgomery
University of Wisconsin, Madison, USA

University of Aberdeen, Scotland

University of California, Berkeley, USA

University of Cambridge, UK

CNRS, Université Paris-Sud, France

University of Michigan, USA

Freie Universität Berlin, Germany

Ruhr-Universität, Germany

University of California, Berkeley, USA

Université de Montréal, Canada

San Francisco State University, USA

Oxford University, UK

University of Virginia, USA

Princeton University, USA

Northwestern University, USA

Harvard University, USA

École Polytechnique Fédérale de Lausanne

University of Southern California, USA
Shigefumi Mori

Raman Parimala

Jonathan Pila

Anand Pillay

Victor Reiner

Peter Sarnak

Joseph H. Silverman

Michael Singer

Vasudevan Srinivas

J. Toby Stafford

Richard Taylor

Ravi Vakil

Michel van den Bergh

Marie-France Vignéras

Kei-Ichi Watanabe

Efim Zelmanov

Shou-Wu Zhang
RIMS, Kyoto University, Japan

Emory University, USA

University of Oxford, UK

University of Notre Dame, USA

University of Minnesota, USA

Princeton University, USA

Brown University, USA

North Carolina State University, USA

Tata Inst. of Fund. Research, India

University of Michigan, USA

Harvard University, USA

Stanford University, USA

Hasselt University, Belgium

Université Paris VII, France

Nihon University, Japan

University of California, San Diego, USA

Princeton University, USA

\section{PRODUCTION}

production@msp.org

Silvio Levy, Scientific Editor

See inside back cover or msp.org/ant for submission instructions.

The subscription price for 2015 is US \$255/year for the electronic version, and $\$ 440 /$ year ( $\$ 55$, if shipping outside the US) for print and electronic. Subscriptions, requests for back issues and changes of subscribers address should be sent to MSP.

Algebra \& Number Theory (ISSN 1944-7833 electronic, 1937-0652 printed) at Mathematical Sciences Publishers, 798 Evans Hall \#3840, c/o University of California, Berkeley, CA 94720-3840 is published continuously online. Periodical rate postage paid at Berkeley, CA 94704, and additional mailing offices.

ANT peer review and production are managed by EditFLOW ${ }^{\circledR}$ from MSP.

\section{PUBLISHED BY}

7 mathematical sciences publishers

nonprofit scientific publishing

http://msp.org/

(C) 2015 Mathematical Sciences Publishers 


\section{Algebra \& Number Theory}

Volume $9 \quad$ No. $2 \quad 2015$

Lifting harmonic morphisms II: Tropical curves and metrized complexes

OMID AMINI, MATTHEW BAKER, ERWAN BRUgallé and JosePH RABINOFF

Noncommutative Hilbert modular symbols

IVAN HOROZOV

$p$-adic Hodge theory in rigid analytic families

REBECCA BELLOVIN

Semistable periods of finite slope families

RUOCHUAN LIU

ANAND DEOPURKAR and ANAND PATEL

Finite-dimensional quotients of Hecke algebras

493

IVAN LOSEV

Semiample invertible sheaves with semipositive continuous hermitian metrics

503 ATSUSHI MORIWAKI 